\title{
Coherent structures generated by inhomogeneities in oscillatory media
}

\author{
Richard Kollár \\ University of Michigan \\ Department of Mathematics \\ 530 Church Street \\ Ann Arbor, MI 48109, USA
}

\author{
Arnd Scheel \\ University of Minnesota \\ School of Mathematics \\ 206 Church Street, S.E. \\ Minneapolis, MN 55455, USA
}

November 10, 2006

\begin{abstract}
We investigate the effect of spatially localized inhomogeneities on a spatially homogeneous oscillation in a reaction-diffusion system. In dimension up to 2 , we find sources and contact defects, that is, the inhomogeneity may either send out phase waves or act as a weak sink. We show that small inhomogeneities cannot act as sources in more than 2 space dimensions. We also derive asymptotics for wavenumbers and group velocities in the far field. The results are established rigorously for radially symmetric inhomogeneities in reaction-diffusion systems, and for arbitrary inhomogeneities in a modulation equation approximation.
\end{abstract}

Keywords: reaction-diffusion, phase diffusion, eikonal equation, coherent structures, target patterns

Running head: Inhomogeneities in oscillatory media

Corresponding author: Arnd Scheel

\section{Oscillatory reaction-diffusion systems}

\subsection{Introduction}

We are interested in patterns that arise in dissipative, spatially extended systems far from equilibrium. The arguably simplest non-equilibrium pattern in a dynamical system is a periodic orbit. Periodic orbits are ubiquitous in dynamical systems, a fact which is partly justified by their robustness. Indeed, when studying ordinary differential equations or partial differential equations posed on bounded domains, periodic orbits are typically robust: the trivial Floquet multiplier associated with the phase of the oscillation is algebraically simple, and for any small perturbation of the system, one will find a nearby periodic orbit, with similar frequency. 
Spatially extended, large systems of oscillators have attracted attention in the physical and mathematical literature in many contexts. A classical prominent example is the BelousovZhabotinsky reaction, a reaction-diffusion system where the chemical concentrations undergo a relaxation-type oscillation which can be sustained for many cycles; see for example [25, 9]. Other examples include biological systems such as cardiac tissue [24], neural systems [23], and ecological systems [6].

When studying such large systems, in unbounded or in large domains, two interrelated issues complicate the concept of a robust oscillation.

First, robustness turns out to be a delicate issue on a technical level. In large domains, the fixed point problem for the Poincare map is ill-conditioned due to clusters of eigenvalues of the linearization near the neutral phase mode; in unbounded domains, the neutral phase mode is even embedded into a continuum of spectrum; see Section 1.3, below. The spatial diffusive coupling, is responsible for this lack of separation between slow phase modes and the fast normal modes near the periodic orbit, since it covers a full band of possible exponential relaxation rates. As a consequence, it is often not obvious if periodic orbits are robust under changes of system parameters!

Second, periodic orbits come in very different spatial flavors: spatially homogeneous oscillations, plane waves, target patterns, and spiral waves, to name but a few. A perturbation theory for spatially extended systems should be able to distinguish between those different types of periodic solutions.

Wave trains, the simplest non-homogeneous periodic solutions, are solutions where the phase $\Phi$ of the oscillation varies periodically in both time and space. Associated with this variation of the phase is the group velocity $c_{\mathrm{g}}$ of a wave train: small, spatially localized variations of the phase $\Phi(t=0)=\Phi_{0}(x)$ are simply advected to leading order, $\Phi(t, x)=\Phi_{0}\left(x-c_{\mathrm{g}} t\right)$. Wave trains come in one-parameter families with parameter $k$, the wavenumber of the spatial variation of the phase. In [17], more general time-periodic solutions of reaction-diffusion systems posed on $x \in \mathbb{R}$ were classified according to their limiting behavior at $x= \pm \infty$. In addition to periodicity, the crucial assumption was convergence to wave trains in the far field. Such solutions, time-periodic and asymptotic to wave trains, were referred to as defects or coherent structures. The crucial property of such solutions turned out to be the sign of the group velocities at the wave trains at $\pm \infty$ : physically, they determine the direction in which perturbations are transported, away or towards the center of the coherent structure; mathematically, they determine Fredholm indices of the linearized period map close to the trivial multiplier 1, and hence give multiplicity results. In summary, the physically relevant "shape", as determined by the group velocities, determines mathematical robustness and multiplicity properties; see Figure 1.1, below for a short summary of the results, connecting transport and multiplicity properties.

The simplest scenario, where the different types of coherent structures emerge, is when a spatially system with a spatially homogeneous oscillation is perturbed by introducing a spatially localized inhomogeneity. It was noted in [17] that wave trains may nucleate at the small inhomogeneity, leading to sources. We pick up this example in this article and study the case of space dimensions two and higher. Our main results characterize the existence of coher- 


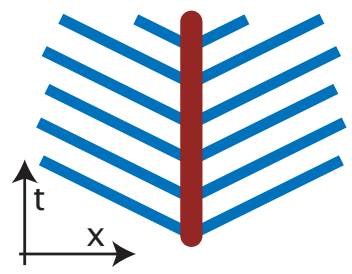

(i) source

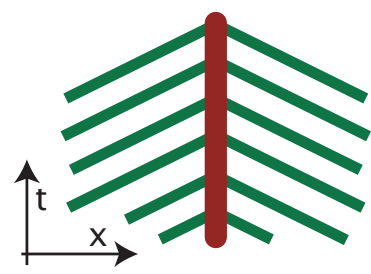

(ii) $\sin \mathrm{k}$

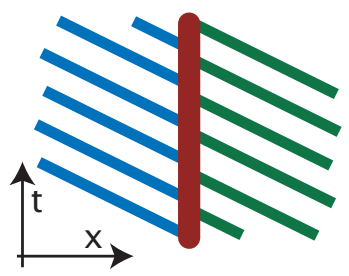

(iii) transmission

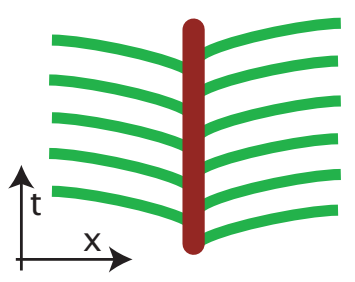

(iv) contact

Figure 1.1: The picture shows schematic space-time plots of the four types of coherent structures that were studied in [17]. The vertical brown bar denotes the location of a defect, where the pattern is not necessarily close to the wave trains. Blue and green lines denote lines of constant phase in the oscillation, with in- and outgoing group velocities, respectively. Sources (i) are isolated, they occur for discrete sets of asymptotic wavenumbers $k_{ \pm}$, group velocities point away from the center. Sinks (ii) come in two-parameter families, group velocities point towards the center, and asymptotic wavenumbers can be prescribed. For transmission defects (iii), group velocities point point in the same direction, $k_{+} k_{-}>0$; only the wavenumber with ingoing group velocity can be prescribed, the wavenumber with outgoing group velocity is selected. For contact defect (iv), the group velocities vanish at infinity. Asymptotic wavenumbers are typically equal.

ent structures, that is, time-periodic solutions which converge to wave trains as $|x| \rightarrow \infty$ for small inhomogeneities. We exhibit a dichotomy similar to the one-dimensional case, between sources, where group velocities point away from the inhomogeneity in the far field, and contact defects, where group velocities point towards the inhomogeneity, but converge to zero as $|x| \rightarrow \infty$. In space dimensions less or equal than two, either sources or contact defects bifurcate, and we give expansions for wavenumbers and group velocities in terms of the strength of the inhomogeneity. In space dimensions larger than two, one only finds contact defects for small inhomogeneities. We prove our results for general reaction-diffusion systems in the case of radially symmetric inhomogeneities. We also formally derive a viscous eikonal equation, for which we prove existence and expansions in the general case, without radial symmetry.

In the remainder of this first section, we will set the scene and explain our main results. The basic setup of oscillations, wave trains, and group velocities is given in 1.2, we define and characterize coherent structures in 1.3 , we give our main results in 1.4, and we conclude with an outline of the remainder of this article.

\subsection{Oscillations, wave trains, and modulations}

As a prototype for non-equilibrium, spatially extended systems, we consider reaction-diffusion systems

$$
u_{t}=D \triangle u+f(u),
$$

$u \in \mathbb{R}^{N}, D=\operatorname{diag}\left(d_{j}\right)>0, f \in \mathbb{C}^{\infty}$, in $x \in \mathbb{R}^{n}$. We assume the existence of an asymptotically stable, spatially homogeneous oscillation. The following list of assumptions roughly states that the spectrum of the period map of (1.1), linearized at a periodic solution, is as stable as possible: it is strictly contained in the unit circle up to a curve of spectrum touching the unit circle at $\lambda=1$ with a quadratic tangency. More precisely, we assume that there exists a 
solution $u(t, x)=u_{*}\left(-\omega_{*} t\right)=u_{*}\left(2 \pi-\omega_{*} t\right)$ to $(1.1)$ and we define

$$
L_{k} u=-D k^{2} u+\omega_{*} u_{\tau}+f^{\prime}\left(u_{*}(\tau)\right) u, \quad L_{k}: \mathcal{D}(L)=H_{\mathrm{per}}^{1}(0,2 \pi) \rightarrow L^{2}(0,2 \pi) .
$$

For stability, we assume that

(i) $\operatorname{spec} L_{k} \cap\left\{\lambda \neq 0 \bmod i \omega_{*}, \operatorname{Re} \lambda \geq 0\right\}=\emptyset$;

(ii) $\operatorname{spec} L_{k} \cap\{\operatorname{Re} \lambda \geq 0\}=\emptyset$ for $k \neq 0$;

(iii) $\lambda=0$ is algebraically simple as an eigenvalue of $\operatorname{spec} L_{0}$;

(iv) the curve of eigenvalues $\lambda(k)$ to $L_{k}$ with $\lambda(0)=0$ satisfies $d=-\lambda^{\prime \prime}(0)>0$.

Examples of such oscillations include all stable periodic solutions to the pure kinetics $u_{t}=$ $f(u)$ if the diffusion matrix is the identity (or close to the identity), an assumption that is typically satisfied for reactions in solvent. It also includes the example of small amplitude oscillations, whenever the Benjamin-Feir stability criterion on sideband instability is satisfied. The chemical oscillations observed in the Belousov-Zhabotinsky reaction provide a prominent experimental example.

Stable homogeneous oscillations are accompanied by a family of wave trains $u_{*}(k \cdot x-\omega t ;|k|)$, which solve

$$
|k|^{2} D u^{\prime \prime}+\omega u^{\prime}+f(u)=0 .
$$

It is not difficult to see that there exists a smooth family of such $2 \pi$-periodic wave train solutions $u_{*}(\cdot ;|k|)$, with $\omega$ given as a function of the parameter $k$. We refer to this dependence $\omega=\Omega(k)$ as the nonlinear dispersion relation. Note that due to isotropy of our medium, $\Omega(k)$ is merely a function of $|k|$. Also note that we slightly abuse notation, writing $u_{*}(\cdot)=u_{*}(\cdot, 0)$ for the spatially homogeneous oscillations. We define the group velocity of a wave train $u_{*}(\cdot ; k)$ via

$$
c_{\mathrm{g}}(k)=\nabla \Omega(k)
$$

Again, by isotropy,

$$
c_{\mathrm{g}}(k)=\left|c_{\mathrm{g}}(k)\right| \frac{k}{|k|}
$$

We emphasize that the existence of wave trains already shows that homogeneous oscillations are not isolated in phase space, and any robustness result needs to take the occurrence of families of solutions into account.

More general solutions can be found by varying the wavenumber of the wave trains on slow spatio-temporal scales. Inserting the Ansatz

$$
u(t, x)=u_{*}\left(-\omega_{*} t-\Phi(T, X) ; \varepsilon \Phi_{X}\right), \quad X=\varepsilon x, \quad T=\varepsilon^{2} t,
$$

into (1.1),we find at order $\varepsilon^{2}$ a viscous eikonal equation

$$
\Phi_{T}=d \Delta_{X} \Phi-\frac{1}{2} \Omega^{\prime \prime}(0)\left|\Phi_{X}\right|^{2}
$$

see for instance [3]. In particular, the nonlinear dispersion relation can be interpreted as a nonlinear flux in a transport equation, and $c_{\mathrm{g}}$ provides precisely the speed of characteristic transport. The viscosity $d$ is defined in item (iv) of our list of linear stability assumptions. 


\subsection{Inhomogeneities and coherent structures}

Our interest here is in the effect of small inhomogeneities in the medium on the oscillations. We therefore consider

$$
u_{t}=D \triangle u+f(u)+\varepsilon g(x, u),
$$

for $\varepsilon$ small, with $g \in C^{\infty}$ smooth and localized, $|g(x, u)|=O\left(|x|^{-2-\beta}\right)$ for $|x| \rightarrow \infty$ and some $\beta>0$.

One may be tempted to analyze this perturbation problem in a large ball of radius $L$ and Neumann boundary conditions, for instance, rather than in unbounded domains. In this case, the Poincaré map possesses an asymptotically stable fixed point which can be readily seen to persist for small values of $\varepsilon$. The validity of this regular perturbation argument in a bounded domain, however, is restricted to $\varepsilon=\mathrm{O}\left(L^{-2}\right)$ at best, since the trivial Floquet exponent is isolated in the spectrum of the period map by a gap of this size only in a large domain.

Our goal in this article is to describe patterns resulting from the introduction of inhomogeneities using perturbation theory in the presence of essential spectrum in unbounded domains. The idealization of the domain as the entire space has the additional advantage of characterizing the resulting patterns by their asymptotic profile in the limit $|x| \rightarrow \infty$.

We distinguish solutions to the inhomogeneous reaction-diffusion system in terms of their transport properties at infinity. In one space-dimension, this task was carried out in a systematic fashion in [17]. We borrow some of the terminology from there and generalize to the multi-dimensional case, next. In [17], we say a solution $u_{\mathrm{c}}(x, t)$ to the (possibly inhomogeneous) reaction-diffusion system is a coherent structure, if $u_{\mathrm{c}}$ is

- time-periodic: $u_{\mathrm{c}}\left(x, t+\frac{2 \pi}{\omega_{\mathrm{c}}}\right)=u_{\mathrm{c}}(x, t)$ for some $\omega_{\mathrm{c}}>0$;

- localized: there are $k_{ \pm}$such that $\left|u_{\mathrm{c}}(x, t)-u_{*}\left(k_{ \pm} x-\omega_{\mathrm{c}} t-\varphi(x) ; k_{ \pm}\right)\right| \rightarrow 0$ for $x \rightarrow \pm \infty$, uniformly in $t$, for some $\varphi(x)$ with $\varphi^{\prime}(x) \rightarrow 0$.

Note that "localized" does not refer to decay, but rather implies convergence as $x \rightarrow \pm \infty$ towards wave trains with certain asymptotic wavenumbers $k_{ \pm}$. We can then classify coherent structures according to the group velocity $c_{\mathrm{g}}\left(k_{ \pm}\right)$at these asymptotic wave trains. We say $u_{\mathrm{c}}$ is a

- source, if $c_{\mathrm{g}}\left(k_{-}\right)<0<c_{\mathrm{g}}\left(k_{+}\right)$;

- $\operatorname{sink}$, if $c_{\mathrm{g}}\left(k_{-}\right)>0>c_{\mathrm{g}}\left(k_{+}\right)$;

- contact defect, if $c_{\mathrm{g}}\left(k_{-}\right)=0=c_{\mathrm{g}}\left(k_{+}\right)$;

- transmission defect, if $c_{\mathrm{g}}\left(k_{-}\right) \cdot c_{\mathrm{g}}\left(k_{+}\right)>0$.

Note that the reflection symmetry of the equation and the uniqueness of the family of wave trains solving (1.3) implies that $\Omega(k)=\Omega(-k)$. The time periodicity of coherent structures implies $\Omega\left(k_{+}\right)=\Omega\left(k_{-}\right)=\omega_{\mathrm{c}}$, which typically, for example if $\Omega^{\prime \prime}(0) \neq 0$, implies that $\left|k_{-}\right|=$ 


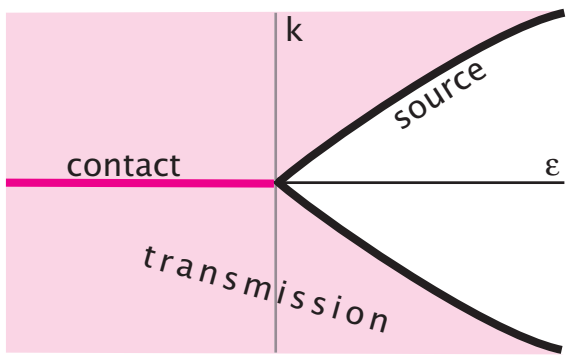

Figure 1.2: Bifurcation diagram for small inhomogeneities in one-dimensional media. Sources exist for positive $\varepsilon$ with emitted wavenumbers $k_{ \pm} \sim \pm \varepsilon$. Contact defects exist for $\varepsilon<0$. The sector between sources and contact defects is filled with transmission defects; see Section 3.2.

$\left|k_{+}\right|$. In $[17, \S 6.5]$, we showed that for small inhomogeneities, there is a dichotomy between the existence of sources and contact defects. A schematic bifurcation diagram is shown in Figure 1.2. The analysis was outlined in [17] but we include a sketch in Section 3.2 here for the convenience of the reader.

In case of radially symmetric defects, the classification is readily adapted. We call a radially symmetric solution $u_{\mathrm{c}}(r, t)$ a coherent structure, if $u_{\mathrm{c}}$ is

- time-periodic: $u_{\mathrm{c}}\left(t+\frac{2 \pi}{\omega_{\mathrm{c}}}, r\right)=u_{\mathrm{c}}(t, r)$ for some $\omega_{\mathrm{c}}>0$;

- localized: there exists $k$ such that $\left|u_{\mathrm{c}}(r, t)-u_{*}\left(k r-\omega_{\mathrm{c}} t-\varphi(r) ; k\right)\right| \rightarrow 0$ for $r \rightarrow \infty$, uniformly in $t$, and $\varphi^{\prime}(r) \rightarrow 0$.

The classification in this radial, multi-dimensional case is somewhat more restrictive: we say $u_{\mathrm{c}}$ is a

- source, if $c_{\mathrm{g}}(k)>0$;

- $\operatorname{sink}$, if $c_{\mathrm{g}}(k)<0$;

- contact defect, if $c_{\mathrm{g}}(k)=0$.

\subsection{Main results: radially symmetric inhomogeneities}

Our main technical results describe coherent structures in spatially homogeneous oscillations generated by radially symmetric inhomogeneities in reaction-diffusion systems. A formal perturbation theory for the periodic solution $u_{*}$ would isolate the time-derivative $u_{*}^{\prime}$ as the kernel in the fixed point equation for the linearized period map (1.1). Similarly, one finds a unique bounded solution $u_{\text {ad }}\left(-\omega_{*} t\right)$ to the adjoint linearized kinetics, $u_{t}=-f^{\prime}\left(u_{*}\right)^{T} u$. We assume that $u_{\text {ad }}$ is normalized such that $\int\left(u_{\text {ad }}(\tau), u_{*}^{\prime}(\tau)\right) \mathrm{d} \tau=1$, where $(\cdot, \cdot)$ denotes the scalar product in $\mathbb{R}^{N}$. Since our perturbation is depending on $x$, we would like to use these eigenfunctions to carry out a perturbation theory in $L^{2}\left(\mathbb{R}^{n}\right)$ or $B C^{0}\left(\mathbb{R}^{n}\right)$, say. If the linearized operator were Fredholm, one would proceed with Lyapunov-Schmidt reduction: one evaluates the perturbation on the kernel and projects it back onto the kernel by taking the scalar product with the 
kernel of the adjoint, and by then normalizing with the scalar product between kernel and cokernel. This procedure fails at the first step: since $u_{*}^{\prime}$ is not localized as a function of $x$, the linearization is not Fredholm; see for example [19, Lemma 6.4]. One may still formally continue to derive an expansion for a perturbed periodic solution by projecting the perturbation, evaluated in the periodic solution, onto the kernel, using the adjoint kernel. However, the fact that the space-time $L^{2}\left(S^{1} \times \mathbb{R}^{n}\right)$-scalar product between $u_{\text {ad }}$ and $u_{*}^{\prime}$ diverges, strongly indicates that the formal results obtained in this fashion will not be valid.

Nevertheless, we define the Melnikov-type coefficient

$M=\left(\operatorname{Vol}\left(S^{n-1}\right)\right)^{-1} \int_{x} \int_{\tau}\left(u_{\mathrm{ad}}(\tau), g\left(|x|, u_{*}(\tau)\right)\right) \mathrm{d} \tau \mathrm{d} x=\int_{0}^{\infty} \int_{\tau}\left(u_{\mathrm{ad}}(\tau), g\left(r, u_{*}(\tau)\right)\right) \mathrm{d} \tau r^{n-1} \mathrm{~d} r$,

which precisely represents the projection of the perturbation on the kernel. As we will see, this coefficient is the essential ingredient to our main result. Our result is stated for radially symmetric solutions, and we therefore may consider the space dimension as a continuous parameter, $n \geq 1$. The theorem describes coherent structures close to the homogeneous oscillation, for $\varepsilon$ small. Close here refers to existence of an appropriate $\delta_{0}$, small, and $\phi=\phi(x)$ such that

$$
\sup _{x}|\nabla \phi(x)|<\delta_{0}, \quad \sup _{x}\left|u(t, x)-u_{*}\left(-\omega_{*} t+\phi(x)\right)\right|_{H_{t}^{1}}<\delta_{0},
$$

where $H_{t}^{1}$ refers to the $H^{1}$-norm with respect to the variable $t \in\left[0,2 \pi / \omega_{*}\right]$.

Notation: We denote by $\mathrm{O}(y)$ and $\mathrm{o}(y)$ the Landau symbols for functions $h$ which vanish at $y=0$ and which satisfy $|h / y| \leq C$ for some $C>0$ and $\lim _{y \rightarrow 0}|h / y|=0$, respectively. We write $\mathrm{o}_{y}(1)$ for functions which converge to zero as $y \rightarrow 0$.

Theorem 1 Consider the reaction-diffusion system (1.7) with $\varepsilon$ small in space dimension $n \leq 2$.

First assume that $\varepsilon M \Omega^{\prime \prime}(0)>0$. Then there exists a constant $\hat{c}$ with $\hat{c} \Omega^{\prime \prime}(0)>0$ such that for all $\varepsilon>0$ sufficiently small, there exists a unique source with emitted wavenumber

$$
\begin{aligned}
& k(\varepsilon)=\hat{c}|M \varepsilon|^{\frac{1}{2-n}}\left(1+\mathrm{o}_{\varepsilon}(1)\right), \quad \text { for } n<2, \\
& k(\varepsilon)=\hat{c} \mathrm{e}^{-\frac{2 d}{\Omega^{\prime \prime} M \varepsilon}}\left(1+\mathrm{o}_{\varepsilon}(1)\right), \quad \text { for } n=2 .
\end{aligned}
$$

In particular, the group velocity in the far-field points outwards.

Next assume that $\varepsilon M \Omega^{\prime \prime}(0)<0$. Then there exists a constant $\hat{c}$ with $\hat{c} \Omega^{\prime \prime}(0)<0$ such that for all $\varepsilon$ sufficiently small, there exists a unique contact defect, that is, the asymptotic wavenumber is $k=0$. At large but finite distances from the center, we have the wavenumber asymptotics

$$
k=\frac{1}{r}\left(\hat{c}+\mathrm{o}_{1 / r}(1)\right), \quad n<2 \text { and } r \gg \log \varepsilon, \quad k=\frac{|\varepsilon M|}{|\varepsilon M| r \log r+r}\left(\hat{c}+\mathrm{o}_{1 / r}(1)\right), \quad n=2 .
$$

In particular, the group velocity converges to zero in the far field but it is pointing inwards at large finite $r$. There also exists a family of sinks with wavenumbers close to 0 for all small $\varepsilon$. There do not exist sources close to the homogeneous oscillations. 

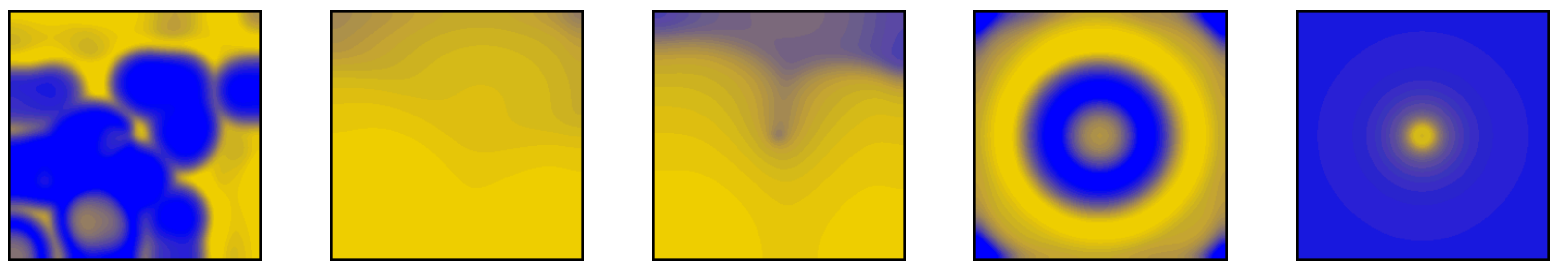

Figure 1.3: Plotted are time snapshots of the inhibitor concentrations $v(x)$ of (1.11). From left to right, a spatially irregular initial condition (left) evolves into a homogeneous oscillation after time $\mathrm{T}=100$ (second) in a homogeneous medium. The next two pictures show how the same initial condition has evolved into a contact defect, $\varepsilon=-0.2$, and a source, $\varepsilon=0.2$. The last picture shows the radially symmetric contact defect, which forms from a homogeneous initial condition, $\varepsilon=-0.2$. Simulations based on EzSPIRAL, [5]. Click on the snapshots to see a movie of the time evolution.

In space dimension $n>2$, there exists a unique branch of contact defects and accompanying sinks, with wavenumber asymptotics

$$
k(r, \varepsilon)=\frac{M \varepsilon}{r^{n-1}}\left(\hat{c}+\mathrm{o}_{1 / r}(1)\right), \quad n>2,
$$

for an appropriate constant $\hat{c} \neq 0$. There do not exist sources regardless of the sign of $\varepsilon M \Omega^{\prime \prime}(0)$.

Remark 1.1 Note that the phase $\phi(r)=\int_{0}^{r} k(s) \mathrm{d} s$ diverges for contact defects for $n \leq 2$, logarithmically in one space-dimension, and very weakly, $\varphi \sim \log \log r$ in two space-dimensions. In higher space-dimensions, the phase converges. In particular, the target sources or target sinks created by inhomogeneities in three space dimensions will have only a finite number of rings, moving outward or inward, depending on the sign of $\varepsilon M \Omega^{\prime \prime}(0)$.

The proof of the theorem will occupy Section 2. Coherent structures solve a degenerate elliptic PDE in $x, t$, with periodic boundary conditions in $t$ and somewhat intricate boundary conditions at $|x|=\infty$. The main difficulty stems from the boundary conditions at infinity, which require the solution to be pointwise in $x$ close to a homogeneous oscillation $u_{*}(\cdot+\phi)$ for some shift $\phi(x)$, where the function $\phi$ and its asymptotics as $|x| \rightarrow \infty$ are to be determined as part of the analysis.

We illustrate the main result with numerical simulations. Figure 1.3 shows snapshots of the dynamics of an oscillatory system in the presence of a localized inhomogeneity. The color coding reflects values of the $v$-component in Barkley's FitzHugh-Nagumo model

$$
u_{t}=\Delta u+\frac{1}{\mu} u(1-u)\left(u-\frac{v+b}{a}\right), \quad v_{t}=\Delta v+u-v+\frac{\varepsilon}{1+|x / 3|^{2}}
$$

with parameters $a=0.3, b=-0.45, \mu=0.095$ on $\Omega=\left\{\left|x_{j}\right| \leq 90\right\}$. The convergence to the final state is much faster in the case of a source.

\subsection{Outline}

The remainder of this article is organized as follows. We introduce spatial dynamics and prove Theorem 1 in Section 2. We rely on an ill-posed dynamical systems formulation, inspired by 
$[8,20]$. The main difficulty is the analysis of an ordinary differential equation in the far-field, which possesses a highly degenerate equilibrium. We unfold the degeneracy using geometric blow-up methods; see $[18,11]$ for a recent account. We then discuss coherent structures which are not necessarily radially symmetric. We therefore derive a viscous eikonal equation that approximates the reaction-diffusion dynamics and discuss shapes of coherent structures in this approximation; Section 3. This discussion largely relies on the Hopf-Cole transformation, which links coherent structures in the eikonal equation to eigenfunctions of Schrödinger operators. We show that coherent structures still are approximately radial in the far field. We conclude Section 3.3 with a discussion of moving inhomogeneities. Main results here again rely on the Hopf-Cole transformation. Coherent structures show more complicated, non-radial patterns such as sonic cones and diffusive profiles. We conclude with a discussion in Section 4 , including a summary of results, discussion of topological and quantitative classifications of defects, and a note on asymptotic stability.

The results on reaction-diffusion systems, Theorem 1, are new. Some of the results on modulation equations in Section 3 appear to be folklore. We refer to [22] and the references therein for related results and an overview of the experiments. We included this a discussion of inhomogeneities in the eikonal approximation since it allows for a comparison with our main technical result, Theorem 1, and for extensions to non-symmetric settings.

Acknowledgments A. Scheel was partially supported by the NSF through grants DMS-0203301 and DMS-0504271. A. Scheel also acknowledges discussions with D. Aronson and O. Zeitouni on properties of Schrödinger operators.

\section{Radial dynamics}

In this section, we prove Theorem 1. Coherent structures solve

$$
-\omega u_{\tau}=D u_{r r}+\frac{n-1}{r} u_{r}+f(u)+\varepsilon g(r, u), \quad \tau \in \mathbb{R} \bmod 2 \pi \mathbb{Z}, r \geq 0,
$$

which we can (formally) rewrite as an abstract first-order differential equation in the radius $r$,

$$
\begin{aligned}
& u_{r}=v \\
& v_{r}=-\frac{n-1}{r} v-D^{-1}\left(\omega \partial_{\tau} u+f(u)+\varepsilon g(r, u)\right) .
\end{aligned}
$$

We consider $(2.1)$ on the Hilbert space $X=H_{\text {per }}^{1 / 2}(0,2 \pi) \times L_{\text {per }}^{2}(0,2 \pi)$. The unbounded principal part of the right-hand side of (2.1) defines a closed operator with domain of definition $X^{1}=$ $H_{\text {per }}^{1}(0,2 \pi) \times H_{\text {per }}^{1 / 2}(0,2 \pi)$. We say $(u, v)(r, \tau)$ is a solution to $(2.1)$ on $J \subset \mathbb{R}_{+}$if $(u, v)(r, \cdot)$ is in $C^{0}(J, X) \cap C^{1}(\operatorname{Int}(J), X) \cap C^{0}\left(\operatorname{Int}(J), X^{1}\right)$. Note that the domain reflects the expected parabolic regularity with two derivatives in $x$ or one derivative in $t$; see $[8,20]$, and [15, Lemma 3.1] for a simple computation justifying this choice of norms.

Note that $(2.1)$ is invariant under the time shift $T_{\phi}:(u, v)(r, \cdot) \mapsto(u, v)(r, \cdot+\phi)$, which therefore maps solutions to solutions. 
The differential equation (2.1) is ill-posed as a dynamical system as its principal part amounts to solving the heat equation as an initial-values problem 'sideways', in $r$.

Our strategy for the proof now consists of several steps. We construct manifolds of bounded solutions close to $\left(u_{*}(\cdot+\varphi), 0\right)$ for $r \in[0, R], W_{-}^{\text {cu }}$, Step 1 , and for $r \in[R, \infty], W_{+}^{\text {cs }}$, Step 2. We then show that these two manifolds intersect transversely along $\left(u_{*}(\cdot+\varphi), 0\right)$, Step 3 . In Step 4, we construct a two-dimensional manifold $W_{+}^{\mathrm{c}}$ that contains this intersection, and we compute the vector field on this manifold in Step 5. Steps 6 and 7 are concerned with an analysis of this vector field, in particular tracking the points on $W_{+}^{\mathrm{c}}$ that yield contact defects, sources, or sinks in $r \geq R$. The analysis involves a geometric blow-up construction, Step 6, and a Dulac map analysis, Step 7. In Step 8, we locate those points in $W_{+}^{\mathrm{c}}$ which yield bounded solutions on $[0, R]$ to leading order in $\varepsilon$. In the final Step 9, we match these bounded solutions on $[0, R]$ with conditions for sources, sinks, and contact defects inside of $W_{+}^{\mathrm{c}}$.

The general strategy is reminiscent of [20], where radially symmetric and time-periodic patterns have been studied close to a time- and space-independent equilibrium of the reactiondiffusion system. We will encounter some additional difficulties in the far field which are similar to the difficulties arising in the study of one-dimensional contact defects; see $[17,18]$.

\section{Step 1: Construction of $W_{-}^{\mathrm{cu}}$}

Proposition 2.1 For all $R>0$, there exists a smooth manifold

$$
W_{-}^{\mathrm{cu}}=\bigcup_{0<r \leq R} W_{-}^{\mathrm{cu}}(r) \times\{r\} \subset X \times \mathbb{R}_{+},
$$

such that $W_{-}^{\mathrm{cu}}(r)$ contains precisely all functions $(u(r), v(r))$ which are boundary values to bounded solutions of (2.1) on $(0, r]$, close to $\left(u_{*}(\cdot+\phi), 0\right)$ in $X$ for some $\phi \in \mathbb{R}$. The manifold is invariant under the action of the temporal shifts $T_{\varphi}$ on $X$, and depends smoothly on $\omega$ and $\varepsilon$ Moreover, the map from solutions to boundary values is a smooth diffeomorphism, equivariant with respect to $T_{\varphi}$.

Proof. The proof is the same as in [20, Prop. 4.7].

\section{Step 2: Construction of $W_{+}^{\text {cs }}$}

We now turn to a description of the bounded solutions at $r=\infty$. We therefore introduce $\alpha=1 / r$ and rewrite $(2.1)$ as

$$
\begin{aligned}
u_{r} & =v \\
v_{r} & =-(n-1) \alpha v-D^{-1}\left(\omega \partial_{\tau} u+f(u)+\varepsilon g(1 / \alpha, u)\right) \\
\alpha_{r} & =-\alpha^{2} .
\end{aligned}
$$

Note that the decay assumption on $g$ implies that $g=\mathrm{O}\left(\alpha^{2+\beta}\right) \in C^{2}$ for $\alpha \geq 0$. In $\alpha=0$, there is a circle of equilibria $u=u_{*}(\cdot+\varphi), v=0$. We are interested in solutions that stay in a vicinity of this circle for all $\alpha$ small. We therefore consider the linearization in one of these 
equilibria

$$
\begin{aligned}
& u_{r}=v \\
& v_{r}=-D^{-1}\left(\omega_{*} \partial_{\tau} u+f^{\prime}\left(u_{*}\right)\right) \\
& \alpha_{r}=0 .
\end{aligned}
$$

This system can be written in the short form $u^{\prime \prime}=-D^{-1} L_{0} u$, so that the spectrum of the righthand side operator in (2.3) is given by the square root of the spectrum of $-D^{-1} L_{0}$ (and the zero eigenvalue from the equation for $\alpha$ ). In particular, there is no purely imaginary spectrum outside of zero since $D^{-1} L_{0} u=\gamma^{2} u$ for some $\gamma \neq 0$ violates our stability assumption (ii). All other eigenvalues come in pairs $(\nu,-\nu)$ by reversibility. The zero eigenvalue is geometrically simple, with kernel spanned by $\left(u_{*}^{\prime}, 0\right)$, again by the stability assumption. Then $\left(0, u_{*}^{\prime}\right)$ provides an obvious generalized eigenfunction. The quadratic expansion of the dispersion relation guarantees that the algebraic multiplicity is not larger than two; see [20].

We therefore have a $2+1$-dimensional center eigenspace $E^{\mathrm{c}}$ consisting of the generalized kernel, and infinite-dimensional stable and unstable eigenspaces $E^{\mathrm{s} / \mathrm{u}}$

Proposition $2.2\left(W_{+}^{\mathrm{cs}}\right)$ There exists a smooth center-stable manifold to the circle of equilibria $\left(u_{*}(\cdot+\varphi), 0\right)$ at $\alpha=0$, tangent to $E^{\mathrm{c}} \oplus E^{\mathrm{s}}$. Moreover, there is a local smooth semi-flow $\Phi_{r}$ on $W_{+}^{\text {cs }}$, and all solutions that stay in a neighborhood of the circle of equilibria for all positive $r \geq R$ are trajectories to this semi-flow on $W_{+}^{\mathrm{cs}}$. Finally, the center-stable manifold is invariant and the flow is equivariant with respect to the action of the symmetry group $T_{\varphi}$.

Proof. The proof is analogous to [17, Thm 3] and will be omitted here.

\section{Step 3: Intersecting $W_{-}^{\mathrm{cu}}$ and $W_{+}^{\mathrm{cs}}$}

By construction, the circle of equilibria at $\left(u_{*}(\cdot+\varphi), 0\right)$ belongs to the intersection of $W_{+}^{\text {cs }}$ and $W_{-}^{\text {cu }}$.

Proposition 2.3 The manifolds $W_{+}^{\mathrm{cs}}$ and $W_{-}^{\mathrm{cu}}$ intersect transversely along $\left(u_{*}(\cdot+\varphi), 0\right)$ in $X$ at any fixed, finite $r \in(0, \infty)$. The intersection $W^{\mathrm{c}}$ is a circle, smoothly depending on $\omega$, $\varepsilon$, and $r$.

Proof. We show that tangent spaces intersect transversely along a one-dimensional subspace. Since the intersection necessarily contains the circle $\left(u_{*}(\tau+\varphi), 0\right)$ at $\varepsilon=0, \omega=\omega_{*}$, this suffices to prove the theorem via Lyapunov-Schmidt reduction.

Transversality is encoded in the immersion map

$$
\iota: T_{*} W_{-}^{\mathrm{cu}}(r) \times T_{*} W_{+}^{\mathrm{cs}}(r) \rightarrow X,\left(\mathbf{u}_{-}^{\mathrm{cu}}(r), \mathbf{u}_{+}^{\mathrm{cs}}(r)\right) \mapsto \mathbf{u}_{-}^{\mathrm{cu}}(r)-\mathbf{u}_{+}^{\mathrm{cs}}(r),
$$

where $\mathbf{u}_{ \pm}^{\mathrm{cu} / \mathrm{cs}}=(u, v)$ solve the linearized equation

$$
\begin{aligned}
& u_{r}=v \\
& v_{r}=-\frac{n-1}{r} v-D^{-1} L_{0} u .
\end{aligned}
$$


Transverse intersection along a one-dimensional manifold is equivalent to $\iota$ being Fredholm of index one with minimal, one-dimensional kernel. From $[20,16]$, we know that $\iota$ is Fredholm and the Fredholm index is given by the relative Morse index at $+\infty$. In order to compute the relative Morse index, we compare the equation to the linearized equation

$$
\begin{aligned}
& u_{r}=v \\
& v_{r}=-\frac{n-1}{r} v-D^{-1} L_{0} u+\lambda u,
\end{aligned}
$$

with spectral parameter $\lambda$. For $\lambda>0$, this equation possesses an exponential dichotomy, the immersion map $\iota$ is Fredholm of index 0 . In the limit $\lambda=0$, there is a double center eigenvalue, which we incorporated in the stable direction $T_{*} W_{+}^{\text {cs }}$. For small positive $\lambda$, these two eigenvalues split in opposite directions. In other words, $T_{*} W_{+}^{\text {cs }}$ is enlarged by one dimension compared to the hyperbolic, Fredholm index 0 situation at $\lambda>0$. A bordering lemma then shows that $\iota$ is Fredholm of index 1. It remains to show that the intersection is transverse, that is, that the kernel of $\iota$ is minimal, one-dimensional.

We can block-diagonalize (2.4) by splitting off the one-dimensional kernel of $D^{-1} L_{0}$ with the associated spectral projection. In this center subspace, we find the unique bounded solution $u=u_{*}^{\prime}(\tau), v=0$. Any linearly independent solution will exhibit a singularity at $r=0$ : indeed, solutions solve $u^{\prime \prime}+\frac{n-1}{r} u^{\prime}=0$, with singularity $r^{2-n}, n \neq 2$, and $\log r, n=2$, for any non-constant solution. In the complement, any solution in the intersection would need to be exponentially localized at infinity. Any element in the kernel of $\iota$ in this hyperbolic component would therefore yield an exponentially localized solution to the system $D \Delta u+f^{\prime}\left(u_{*}(\tau)\right) u+$ $\omega_{*} \partial_{\tau} u=0$. In particular, the Fourier transform $\hat{u}(k, \tau)$ of this exponentially localized solution would be smooth in Fourier space and contribute to the kernel of $L_{k}$. Since all $L_{k}$ with $k \neq 0$ are invertible by assumption, this implies $\hat{u} \equiv 0$, and concludes the proof.

\section{Step 4: Extending the intersection $-W_{+}^{c}$}

The intersection $W^{\text {c }}$ typically crosses the boundary of $W_{+}^{\text {cs }}$ for $r \rightarrow \infty$, that is, solutions do not stay close to $u_{*}$ for $r \rightarrow \infty$ for arbitrary parameters $\varepsilon$ and $\omega$. The construction of the center-stable manifold $W_{+}^{\text {cs }}$ incorporates all solutions with mild growth. In order to single out the solutions that actually stay bounded as $r \rightarrow \infty$, we will analyze the flow in the center direction at $r=\infty$ more carefully. We therefore construct a center manifold $W_{+}^{\mathrm{c}} \subset W_{+}^{\text {cs }}$, which is tangent to $E^{\mathrm{c}}$ at $r=\infty$, and contains $W^{\mathrm{c}}$.

Proposition 2.4 There exists a $2+1$-dimensional smooth center-manifold near the circle of equilibria $(u(\cdot+\varphi), 0)$ at $\alpha=0$, which is tangent to $E^{\mathrm{c}}$, and contains all solutions that are bounded on $(0, \infty)$,

$$
W^{\mathrm{c}} \subset W_{+}^{\mathrm{c}} \subset W_{+}^{\mathrm{cs}}
$$

for sufficiently large $r$.

Proof. The proof is analogous to [20, Thms 3.7,4.8]. 


\section{Step 5: The vector field on $W_{+}^{\mathrm{c}}$}

Invariance of $W_{+}^{\mathrm{c}}$ together with the condition on tangency allows us to compute the Taylor expansion of the reduced vector field. We denote eigenvectors and adjoint eigenvectors in the generalized kernel by

$$
e_{0}=\left(u_{*}^{\prime}, 0\right), \quad e_{1}=\left(0, u_{*}^{\prime}\right), \quad e_{0}^{*}=\left(D u_{\mathrm{ad}}, 0\right), \quad e_{1}^{*}=\left(0, D u_{\mathrm{ad}}\right),
$$

where $L_{0}^{*} u_{\text {ad }}=0$, and we normalize $\left(D u_{\text {ad }}, u_{*}^{\prime}\right)=1$, so that $\left(e_{i}, e_{j}^{*}\right)=\delta_{i j}$. We introduce coordinates on the center manifold by parameterizing the tangent bundle of the circle of equilibria with $\theta$, the symmetry action, and $\kappa$ :

$$
(u, v)=\left(u_{*}^{\prime}(\cdot+\theta), 0\right)+\kappa e_{1}(\cdot+\theta)+\psi[\kappa](\cdot+\theta),
$$

where $\psi$ denotes the (symmetry-invariant) graph of the center manifold, $\left.\left(e_{j}^{*}(\cdot), \psi(\cdot)\right)\right)=0$. The equation on the center manifold is independent of $\theta$, by symmetry, and it therefore suffices to track the $\kappa$ - and $\alpha=1 / r$-dependence of the vector field. A straightforward expansion shows that we necessarily recover the phase diffusion equation (1.6) at second order:

$d\left(\kappa^{\prime}+(n-1) \alpha \kappa\right)=\left(\omega-\omega_{*}\right)+\frac{1}{2} \Omega^{\prime \prime}(0) \kappa^{2}+\mathrm{o}\left(|\kappa|^{2}+|\alpha|^{2}\right)+\mathrm{O}\left(\left|\omega-\omega_{*}\right|\left(\left.|\kappa|^{2}|+| \alpha\right|^{2}\right)\right), \quad \alpha^{\prime}=-\alpha^{2}$.

The $\varepsilon$-dependent terms only contribute to the higher-order terms because of the rapid decay of $g$.

\section{Step 6: Geometric blow-up}

The vector field on $W_{+}^{\mathrm{c}}$ possesses a doubly degenerate equilibrium at $\alpha=\kappa=0$ : the linearization at this equilibrium vanishes, leading order terms are quadratic in $\alpha$ and $\kappa$. This degeneracy is unfolded by the parameter $\omega$. Varying $\omega$, the equilibrium undergoes a saddlenode bifurcation inside $\alpha=0$. It turns out that we are interested in connections to the unstable equilibrium in this saddle-node bifurcation. In order to track its stable manifold, we invoke a desingularization method, the geometric blow-up [4, 11]. In fact, the system on the center manifold is similar to the system studied in [18], to which we will refer for more details on the construction. The similarity to the eigenvalue problem for the $n$-dimensional Laplacian in [18] comes as no surprise as the formal eikonal long-wavelength approximation is conjugate to the linear heat equation via the Hopf-Cole transformation.

We briefly summarize the blow-up construction from [18]. To leading order, the system (2.5) is homogeneous, quadratic, with variables $(\kappa, \alpha, \delta) \in \mathbb{R}^{3}$, once we set $\omega-\omega_{*}= \pm d \delta^{2}$. We introduce polar coordinates $\mathbb{R}_{+} \times S^{2} \mapsto \mathbb{R}^{3}$, thus blowing up the origin into a 2 -sphere, and then introduce local coordinates corresponding to stereographic projections. More explicitly, these coordinates are

$$
\begin{array}{rll}
\alpha_{1}=\alpha, & \kappa_{1}=\kappa / \alpha, & \delta_{1}=\delta / \alpha ; \\
\alpha_{2}=\alpha / \delta, & \kappa_{2}=\kappa / \delta, & \delta_{2}=\delta .
\end{array}
$$

In the new coordinates, after rescaling time with the Euler multipliers $\alpha_{1}$ and $\delta_{2}$, respectively, the equations read

$$
\kappa_{1}^{\prime}=-(n-2) \kappa_{1}+\frac{\Omega^{\prime \prime}}{2 d} \kappa_{1}^{2} \pm \delta_{1}^{2}+\mathrm{o}_{\alpha_{1}}(1)
$$




$$
\begin{aligned}
\alpha_{1}^{\prime} & =-\alpha_{1} \\
\delta_{1}^{\prime} & =\delta_{1}
\end{aligned}
$$

and

$$
\begin{aligned}
\kappa_{2}^{\prime} & =-(n-1) \alpha_{2} \kappa_{2}+\frac{\Omega^{\prime \prime}}{2 d} \kappa_{2}^{2} \pm 1+\mathrm{o}_{\delta_{2}}(1) \\
\alpha_{2}^{\prime} & =-\alpha_{2}^{2} \\
\delta_{2}^{\prime} & =0 .
\end{aligned}
$$

In the following, we will assume $\Omega^{\prime \prime}>0$ and choose the '-'-sign in the two equations. The other case is completely analogous. We depicted the phase portrait in this rescaled time in Figure 2.1. Note that with the convention $\Omega^{\prime \prime}>0$, the equilibria with $\kappa>0$ correspond to asymptotically positive, outward pointing group velocity and connections to those equilibria are the sources we are seeking.

\section{Step 7: The Dulac map}

The stable manifold of the family of saddles in the 2-chart can be continued into the 1-chart until it enters the section $\alpha=1 / R$. Its location in this section can be computed as follows. The stable manifold enters a neighborhood of the singular equilibrium $\kappa_{1}=\delta_{1}=\alpha_{1}=0$ along the weak unstable (or center) direction from the negative $\kappa$ direction. It will leave the neighborhood of this equilibrium along the stable $\alpha$-direction. We start by computing the transition map for the approximation

$$
\begin{aligned}
& \kappa_{1}^{\prime}=\frac{\Omega^{\prime \prime}}{2 d} \kappa_{1}^{2}, \quad \alpha_{1}^{\prime}=-\alpha, \quad \delta_{1}^{\prime}=\delta_{1}, n=2, \quad \text { or } \\
& \kappa_{1}^{\prime}=-(n-2) \kappa_{1}, \quad \alpha_{1}^{\prime}=-\alpha, \quad \delta_{1}^{\prime}=\delta_{1}, n<2 .
\end{aligned}
$$

In the section $\kappa_{1}=m, m>0$ small, the distance of the stable manifold to the singular sphere can be expanded as $\alpha_{\text {in }}=c_{\alpha} \delta+\mathrm{O}\left(\delta^{2}\right)$. We want to compute the location of this manifold after passage near the singular equilibrium, when it hits the section $\alpha_{1}=m$. Since the flow in the direction of $\alpha_{1}$ is linear, we find the time of flight as $T=\log \delta+\mathrm{O}(1)$, with error terms smooth in $\delta$. In order to compute the $\kappa_{1}$-coordinate after time $T$, we need to compute the flow $\Phi_{T}$ in the $\kappa$-direction, with initial condition $\kappa_{1}=m$. We find

$$
\begin{array}{ll}
\Phi_{T}(m)=\mathrm{e}^{-(n-2) T} m, & \text { for } n<2 \\
\Phi_{T}(m)=\frac{1}{\frac{1}{m}-\frac{\Omega^{\prime \prime}}{2 d} T}, & \text { for } n=2,
\end{array}
$$

which gives

$$
\begin{aligned}
& \kappa_{1}=\Phi_{T}(m)=\delta^{2-n} m+\mathrm{O}\left(\delta^{2(2-n)}\right), \quad \text { for } n<2 \\
& \kappa_{1}=\Phi_{T}(m)=-\frac{2 d}{\Omega^{\prime \prime} \log \delta}+\mathrm{O}\left(\frac{1}{(\log \delta)^{2}}\right), \quad \text { for } n=2 .
\end{aligned}
$$

In order to estimate the influence of the error terms, we set $\kappa_{1}=\kappa_{1}^{*}+\hat{\kappa}_{1}$, where $\kappa_{1}^{*}$ is the above approximation, with the exact initial conditions, $\hat{\kappa}_{1}(t=T)=0$. A straightforward fixed point argument then shows that

$$
\hat{\kappa}_{1}(t)=\mathrm{O}\left(t^{-2}\right), \text { for } n=2, \quad \hat{\kappa}_{1}(t)=\mathrm{O}\left(\mathrm{e}^{-(2-n+\beta)|t|}\right), \text { for } n<2 .
$$



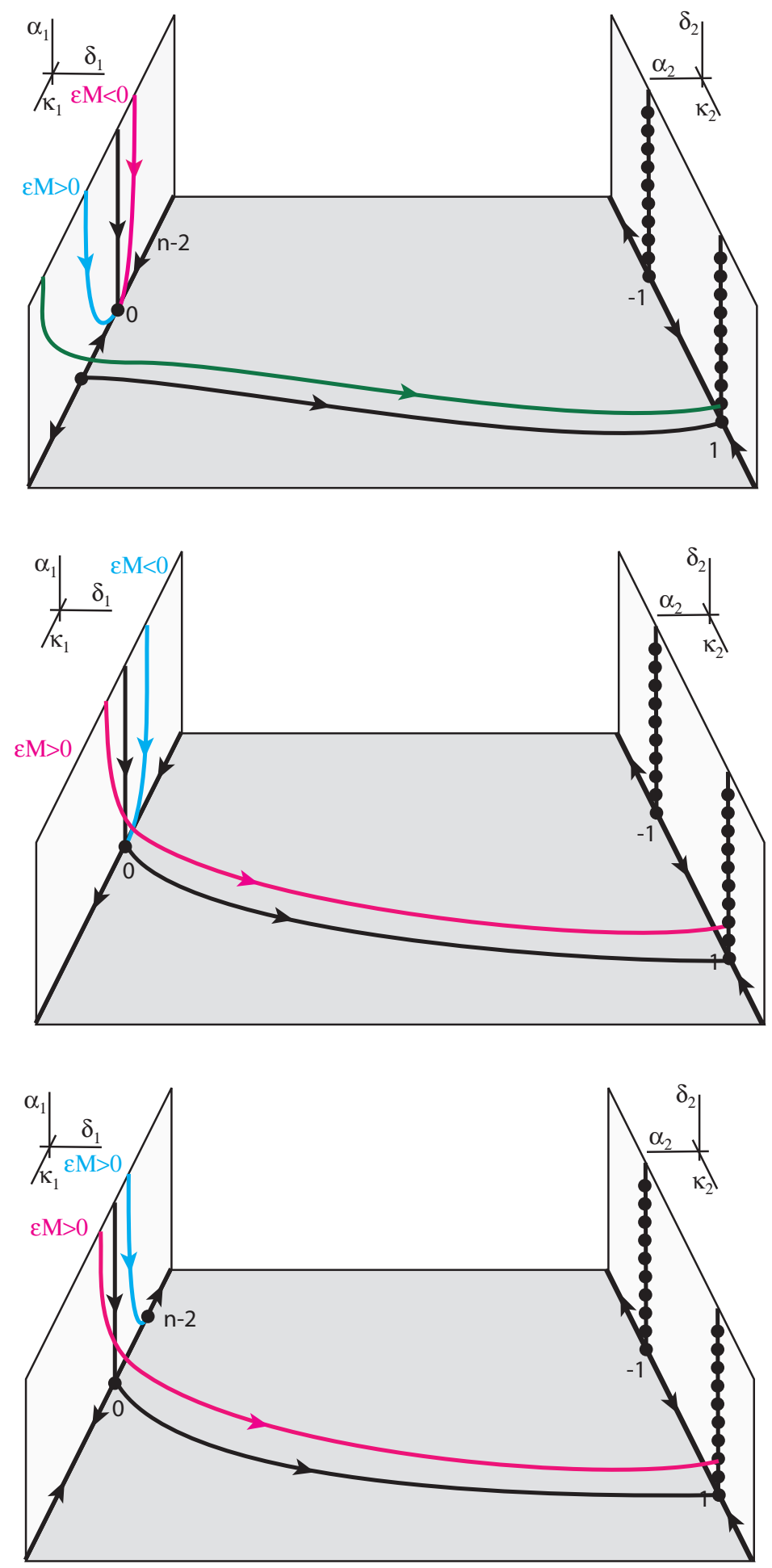

Figure 2.1: The connecting orbits between $r=0$ and the 1-chart and from the 1-chart to the 2-chart, for $n>2$, top figure, $n=2$, middle figure, and $n<2$, bottom figure. The entering trajectory in $\delta_{1}=0, \alpha_{1}>0$ are obtained from matching with the region $r \leq R$. We set $\Omega^{\prime \prime} / 2 d=1$ in all cases. 
This shows that the expansions (2.6) are valid for the full system incorporating the error terms, as well.

\section{Step 8: The Melnikov integral}

In order to conclude the construction, we have to match the stable manifold of the asymptotic

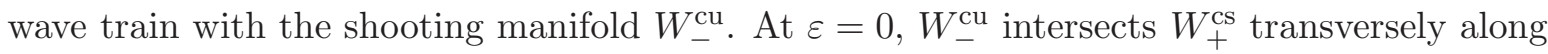
$W^{\mathrm{c}} \subset W_{+}^{\mathrm{c}}$. It is therefore sufficient to compute the location of $W_{-}^{\mathrm{cu}} \cap W_{+}^{\mathrm{c}}$ and then propagate the solution to $\alpha=0$. To leading order in $\delta$, the location of $W^{\mathrm{c}}$ remains unchanged since $\delta$ enters the equation only at quadratic order. To leading order in $\varepsilon$, the location can be computed by solving the variational problem following the standard procedure in heteroclinic bifurcations as follows. We denote by $\Phi(\rho, \sigma)$ the evolution operator to the linearized equation

$$
\begin{aligned}
& u_{\rho}=\mathrm{e}^{\rho} v \\
& v_{\rho}=-(n-1) v-\mathrm{e}^{\rho} D^{-1} L_{0} u,
\end{aligned}
$$

which can be readily constructed using the exponential dichotomies at $r=\infty$ and $r \sim 0$. Similarly, we can define the adjoint operator $\Phi^{*}(\rho, \sigma)$ as solution to

$$
\begin{aligned}
& u_{\rho}=\mathrm{e}^{\rho} L_{0}^{*} D^{-1} v \\
& v_{\rho}=-\mathrm{e}^{\rho} u+(n-1) v .
\end{aligned}
$$

We are interested in the location $\partial_{\varepsilon} \kappa_{-}(R)$, the derivative of the manifold $W^{\mathrm{c}}$ in the direction

of $e_{1}$, which is found by taking the scalar product of the perturbation, integrated along the linearized flow, with $e_{1}^{*}$,

$$
\begin{aligned}
\partial_{\varepsilon} \kappa_{-}(R) & =\left\langle e_{1}^{*}, \int_{-\infty}^{\mathrm{e}^{R}} \Phi\left(\mathrm{e}^{R}, \sigma\right) \mathrm{e}^{\sigma}\left(0,-D^{-1} \partial_{\varepsilon} g\left(\mathrm{e}^{\sigma}, u_{*} ; 0\right)\right) \mathrm{d} \sigma\right\rangle \\
& =-\int_{-\infty}^{\mathrm{e}^{R}}\left\langle\Phi^{*}\left(\mathrm{e}^{R}, \sigma\right) e_{1}^{*},\left(0, D^{-1} \partial_{\varepsilon} g\left(\mathrm{e}^{\sigma}, u_{*} ; 0\right)\right) \mathrm{e}^{\sigma}\right\rangle \mathrm{d} \sigma .
\end{aligned}
$$

An explicit computation shows that

$$
\Phi^{*}\left(\mathrm{e}^{R}, \sigma\right) e_{1}^{*}=\left(0, \mathrm{e}^{(n-1) \sigma} D u_{\mathrm{ad}}\right),
$$

which then gives

$$
\begin{aligned}
\partial_{\varepsilon} \kappa_{-}(R) & =-\int_{-\infty}^{\mathrm{e}^{R}}\left\langle D u_{\mathrm{ad}}, D^{-1} \partial_{\varepsilon} g\right\rangle \mathrm{e}^{n \sigma} \mathrm{d} \sigma \\
& =-\int_{0}^{R}\left\langle u_{\mathrm{ad}}, \partial_{\varepsilon} g\right\rangle r^{n-1} \mathrm{~d} r \\
& =-\left(\operatorname{Vol}\left(S^{n-1}\right)\right)^{-1} \int_{\mathbb{R}^{n}} \int_{0}^{2 \pi}\left(u_{\mathrm{ad}}(\tau), \partial_{\varepsilon} g\left(|x|, u_{*}(\tau) ; 0\right)\right) \mathrm{d} \tau \mathrm{d} x+\mathrm{o}_{1 / R}(1) .
\end{aligned}
$$

\section{Step 9: Matching core and far field}

We first consider the case of space dimension $n>2$. At $\delta=0$, the heteroclinic given by $u_{*}(\tau)$ connects to a sink in the singular blow-up chart. The connection is therefore robust 
under $\varepsilon$-perturbations, yielding a robust family of contact defects, asymptotic wavenumber zero. Unfolding in $\delta$, a simple transitivity lemma shows that orbits pass near the equilibrium $\kappa_{1}=0$ in the singular chart and then connect to the sink in the 2-chart, corresponding to sinks with asymptotically negative group velocity in the reaction-diffusion system. We will derive expansions for the wavenumber below, which will then prove Theorem 1 in the case $n>2$.

In the case $n \leq 2$, the heteroclinic connects to a saddle and the above Melnikov analysis shows that turning on $\varepsilon$ actually breaks the connection. For $\partial_{\varepsilon} \kappa_{-}(R)>0$, we find a connection to the sink $\kappa_{1}=0$ in the one-chart, thus yielding contact defects. Again, nonzero values of $\delta$ yield connections to the sink in the 2-chart and thereby accompanying sinks in the full reaction-diffusion system. In the case $\partial_{\varepsilon} \kappa_{-}(R)<0$, we find an intersection with the stable manifold of the saddle in the 2-chart, whose location at $\alpha=1 / R$ is $\kappa=M \varepsilon+\mathrm{O}\left(\varepsilon^{2}\right)$. We match this expansion with the expansion (2.6) from the singular chart and solve for $\delta^{2-n}$ or $\mathrm{e}^{-1 / \delta}$, respectively with the implicit function theorem. We find the expansion

$$
\begin{aligned}
\delta & =c_{*}|M \varepsilon|^{1 /(2-n)}\left(1+\mathrm{o}_{\varepsilon}(1)\right), \quad \text { for } n<2, \\
\delta & =c_{*} \mathrm{e}^{-\frac{2 d}{\Omega^{\prime \prime} M \varepsilon}}\left(1+\mathrm{o}_{\varepsilon}(1)\right), \quad \text { for } n=2,
\end{aligned}
$$

for some constants $c_{*}$ with $c_{*} \Omega^{\prime \prime}>0$. Next, we replace $\delta$ by $k$, using the relations $\omega-\omega_{*}=\delta^{2}$ and $\omega-\omega_{*}=\Omega^{\prime \prime} k^{2} / 2+\mathrm{O}\left(k^{4}\right)$, so that

$$
\begin{aligned}
k & =\hat{c}(M \varepsilon)^{1 /(2-n)}\left(1+\mathrm{o}_{\varepsilon}(1)\right), \quad \text { for } n<2, \\
k & =\hat{c} \mathrm{e}^{-\frac{2 d}{\Omega^{\prime \prime} M \varepsilon}}\left(1+\mathrm{o}_{\varepsilon}(1)\right), \quad \text { for } n=2 .
\end{aligned}
$$

This proves the existence of sources in the case $n \leq 2$ and the expansion for the asymptotic wavenumber. Again, there are also accompanying sinks with asymptotic wavenumbers $|k|>$ $\left|k_{\text {source }}(\varepsilon)\right|$. This proves existence and asymptotics for sources in Theorem 1 in the case $n \leq 2$.

The existence and asymptotics for contact defects, $\kappa=0$, are simpler. The connection is robust in the 1-chart, with asymptotics

$$
\kappa_{1} \rightarrow \frac{2 d(2-n)}{\Omega^{\prime \prime}}, \quad n<2, \quad \kappa_{1} \sim \frac{1}{\log r+(\varepsilon M)^{-1}}, \quad n=2, \quad \text { and } \quad \kappa_{1} \sim r^{2-n}, \quad n>2,
$$

which leads to wavenumber asymptotics

$$
k \sim \frac{2 d(2-n)}{\Omega^{\prime \prime} r}, \quad n<2, \quad k \sim \frac{\varepsilon M}{\varepsilon M r \log r+r}, \quad n=2, \quad \text { and } \quad k \sim M \varepsilon r^{1-n}, \quad n>2 .
$$

Remark 2.5 The analysis presented here simplifies considerably when studying systems with a gauge symmetry, such as $\lambda-\omega$ systems or Ginzburg-Landau equations. The gauge symmetry in these systems is represented as an action $T$ of the circle group $\phi \in S^{1}$, so that $u$ is a solution if and only if $T_{\phi} u$ is. The simplest periodic solutions then are equilibria with respect to this action, $u(t, x)=T_{k x-\omega(k) t} u_{*}(k)$. Coherent structures can be found as solutions of the form $u(t, x)=$ $T_{-\omega t} u_{*}(x)$, so that $u_{*}$ satisfies a (time-independent) elliptic equation with a free parameter $\omega$. In the radially symmetric case, this reduces the problem to an ordinary differential equation; see [10] for an analysis of such a problem. For non-radially symmetric inhomogeneities, the resulting time-independent PDE problem is similar to the radially symmetric case, without gauge symmetry, that we analyzed here. 


\section{The eikonal approximation}

In this section, we derive the viscous eikonal equation formally from the reaction-diffusion system and analyze profiles of coherent structures in this approximation. We find results similar to the results in Theorem 1, and we are able to discuss non-radially symmetric inhomogeneities, and the effect of moving inhomogeneities. The results differ from the results in the previous section since they assume a particular spatial scaling of the inhomogeneity for small amplitude $\varepsilon$. This special scaling is consistent with the modulation Ansatz, albeit not typical in a given system. We also emphasize that this formal approach yields flat $\mathrm{e}^{-1 / \varepsilon}$-expansions, while the approximation itself is only correct to order $\varepsilon^{2}$. It is therefore not clear at all, why these formal results give similar asymptotics as the ones that we rigorously obtained in the previous section.

\subsection{Stationary non-symmetric inhomogeneities}

We consider

$$
u_{t}=D \triangle u+f(u)+\varepsilon^{2} g(\varepsilon x, u),
$$

with $\varepsilon$ small. We follow the derivation in $[3, \S 4.3]$, where the computations are shown for a wave train with nonzero spatial wave number. We substitute the Ansatz (1.5)

$$
u(t, x)=u_{*}\left(\Phi(T, X)-\omega t ; \varepsilon \nabla_{X} \Phi(T, X)\right)+\varepsilon^{2} u_{1}(-\omega t, T, X) ; \quad X=\varepsilon x, T=\varepsilon^{2} t,
$$

into (1.7) and expand in powers of $\varepsilon$. At order $\varepsilon^{2}$, we find after a short computation

$$
\Phi_{T} u_{*}^{\prime}-\Delta_{X} \Phi D u_{*}^{\prime}-\left|\nabla_{X} \Phi\right|^{2} D u_{*}^{\prime \prime}-g\left(X, u_{*}\right)=L_{0} u_{1}(x) .
$$

Solvability requires that the left side of (3.3) belongs to the range of $L_{0}$. We denote by $u_{\text {ad }}$ the kernel of the $L^{2}$-adjoint of $L_{0}$ with normalization $\left(u_{\mathrm{ad}}, u_{*}^{\prime}\right)=1$. After some calculations, this solvability condition turns out to be equivalent to

$$
\Phi_{T}=d \Delta_{X} \Phi-\frac{1}{2} \Omega^{\prime \prime}\left|\nabla_{X} \Phi\right|^{2}+\bar{G}(X)
$$

where

$$
\bar{G}(X)=\int_{0}^{2 \pi}\left(g\left(X, u_{*}(\zeta)\right), u_{\text {ad }}(\zeta)\right) \mathrm{d} \zeta
$$

see also [3] for a similar expansion. The Hopf-Cole transformation

$$
A=\mathrm{e}^{a \Phi}, \quad a=-2 d / \Omega^{\prime \prime},
$$

linearizes the eikonal equation so that we find

$$
A_{T}=d \Delta A+\bar{G}(X) A .
$$

Coherent structures are solutions with $\Phi_{T}=-\omega$, which is equivalent to $A_{T}=-\omega A$. They therefore correspond to eigenfunctions of the Schrödinger eigenvalue problem

$$
-\omega A=d \Delta A+\bar{G}(X) A .
$$


For $n=1,2$, expansions on the leading eigenvalue have been derived in many contexts, going back to Landau [12] and Simon [21]. For instance, for $d=1$, [21, Thm 3.4] states that

$$
\omega \sim \mathrm{e}^{-\frac{4 \pi}{\int G}}
$$

for small $\int G$, which agrees with our expansion in Theorem 1. To see this, replace $\omega=\Omega^{\prime \prime} k^{2}$ in (3.7), solve for $k$, and recall that $M=\int G / \operatorname{Vol}\left(S^{1}\right)=\int G / 2 \pi$.

From a phenomenological viewpoint, we are most interested in the asymptotics of the wave number in the far field, that is, in $\nabla \Phi(X)$ as $X \rightarrow \infty$. In the Schrödinger formulation, this amounts to computing decay and growth properties of the eigenfunction in the far field. For instance, assume that an eigenfunction $A$ possesses exponential asymptotics $\mathrm{e}^{-\gamma|x|}$ for some $\gamma \in \mathbb{R}$. We can then infer phase asymptotics for $\Phi=\frac{1}{a} \log A$,

$$
\Phi \sim \frac{1}{2 d} \Omega^{\prime \prime} \gamma|x|,
$$

hence $\operatorname{sign} k=\operatorname{sign} \Omega^{\prime \prime} \gamma$. In particular, exponential decay generates outward group velocities and exponential growth inward group velocities. The exponential localization of eigenfunctions outside of the essential spectrum yields sources in the eikonal equation.

For $n>2$ the nonexistence of eigenvalues (point spectrum) for small potential follows readily from Hardy's inequality [13], which states that for any $f$, with $f^{\prime}$ integrable,

$$
\int_{0}^{\infty}|f(x) / x|^{p} \mathrm{~d} x<\left(\frac{p}{p-1}\right)^{p} \int_{0}^{\infty}\left|f^{\prime}(x)\right|^{p} \mathrm{~d} x
$$

Indeed, for $n=3$, say, and $\bar{G}$ radial, the symmetric form associated with the elliptic operator is positive whenever $\bar{G}<\frac{d}{4 r^{2}}$ :

$$
\int_{\mathbb{R}^{3}}\left(d|\nabla A|^{2}-\bar{G}(x) A^{2}\right)|x|^{2} \mathrm{~d} x \geq 4 \pi d \int_{0}^{\infty}\left(r^{2} A_{r}^{2}-\frac{1}{4} A^{2}\right) \mathrm{d} r
$$

and by Hardy's inequality

$$
\int_{0}^{\infty} r^{2} A_{r}^{2} \mathrm{~d} r=\int_{0}^{\infty}\left((r A)_{r}^{2}-A^{2}-\left(A^{2}\right)^{\prime} r\right) \mathrm{d} r=\int_{0}^{\infty}(r A)_{r}^{2} \mathrm{~d} r \geq \int_{0}^{\infty} \frac{1}{4} A^{2} \mathrm{~d} r .
$$

Note however that in the scaling used to derive the eikonal approximation, $\bar{G}$ need not be small. In particular, potentials which are small in amplitude but long-range in the sense imposed by the scaling can create sources.

The following result appears to be standard, but we were unable to locate a good reference and therefore include a proof in the appendix.

Proposition 3.1 Consider the eigenvalue problem (3.6) in $\mathbb{R}^{2}$ with $d=1$ and smooth potential $\bar{G}$ with

$$
\left.\mid \bar{G}(r, \varphi))|+| \partial_{r} \bar{G}(r, \varphi)|+| \partial_{\varphi} \bar{G}(r, \varphi)\right) \mid=\mathrm{O}\left(r^{-1-\beta}\right)
$$

in polar coordinates, as $r=|x| \rightarrow \infty$, for some $\beta>0$. Let $u \in C^{2}$ be a positive eigenfunction to an eigenvalue $\omega=-\gamma^{2}<0$. 
We then have the following renormalized asymptotics with $\tilde{A}(r, \varphi)=\sqrt{r} \mathrm{e}^{\gamma r} A(r, \varphi)$ :

$$
\left|\tilde{A}(r, \cdot)-\tilde{A}_{\infty}(\cdot)\right|_{H^{1}} \rightarrow 0,
$$

for some $\tilde{A}_{\infty}(\cdot) \in H_{\text {per }}^{1}(0,2 \pi), \tilde{A}_{\infty} \neq 0$.

There is a tremendous amount of literature on decay properties of solutions to elliptic equation; see for instance the early references [1] for upper bounds, and [2, Thm 3.2], for lower bounds. The decay properties for $\bar{G}$ needed here are weaker than the decay properties in the analysis of small potentials. For instance, long-range potentials, $\bar{G} \sim|x|^{-1-\beta}, \beta<1$, create infinitely many bound states, while the short range potentials from Theorem 1 create a unique bound state at small amplitude. The proposition states that the long range of the potential does not influence the decay rate of the eigenfunction at leading order.

We expect that contact defects are highly non-unique in the non-radially symmetric case: we expect an infinite-dimensional manifold, corresponding to different asymptotic azimuthal profiles. Asymptotics for this situation do not appear to be well understood.

From Proposition 3.1, we can conclude that level lines of the phase are corrections to increasingly large circles. Rings sent out by the sources do not converge to circles, the deviation will remain $\mathrm{O}(1)$. Indeed, in the far field, level sets of the phase $\Phi=\log A=C$ solve

$$
\log \tilde{A}_{\infty}(\varphi)+\gamma r+\frac{1}{2} \log r=C,
$$

so that with the formal inverse $h\left(\gamma r+\frac{1}{2} \log r\right)=r$, we have the asymptotic parametrization $r=h\left(C-\log \tilde{A}_{\infty}(\varphi)\right)$ of level sets. Since $h^{\prime} \sim 1 / \gamma$ for large $r, r$ will not be constant on level sets if $\tilde{A}_{\infty}$ is not constant.

Note however that the wavenumber does converge to $k x /|x|$, since azimuthal gradients decay as $1 /|x|$.

\subsection{Transmission, sinks, and contacts}

Deviating from our primary focus on higher-dimensional coherent structures, we recall results on transmission defects in one-dimensional media from [17], preparing for the analysis of moving inhomogeneities in the next section. Our interest is in the response of the inhomogeneity to incoming wave trains. A fairly complete answer can be provided in one space dimension.

We start with the viscous eikonal equation, $d=1, \Omega^{\prime \prime}=1$ and a localized inhomogeneity:

$$
-\omega=\Phi_{x x}-\frac{1}{2} \Phi_{x}^{2}+\varepsilon G(x)
$$

which we write as a non-autonomous first-order ODE

$$
\Phi_{x}=k, \quad k_{x}=-\omega+\frac{1}{2} k^{2}-\varepsilon G(x) .
$$

Note that the equation for $k$ is independent of $\Phi$ and can therefore be solved independently,

$$
k_{x}=-\omega+\frac{1}{2} k^{2}-\varepsilon G(x) .
$$


At $x= \pm \infty$, the inhomogeneity disappears, and we find

$$
k_{x}=-\omega+\frac{1}{2} k^{2} .
$$

This equation possesses equilibria $k= \pm \sqrt{2 \omega}$ for $\omega>0$ corresponding to the planar wave trains, equilibria $k=0$ for $\omega=0$ corresponding to the homogeneous oscillations, and no bounded solutions for $\omega<0$. As a consequence, bounded solutions to (3.10) exist only for $\omega \geq 0$. As $x \rightarrow+\infty$, there exists a unique solution which converges to $k=\sqrt{2 \omega}$. All other bounded solutions converge to $k=-\sqrt{2 \omega}$.

Let $W_{+}^{\mathrm{s}}=\left(-\infty, k_{+}^{*}\right)$ be the set of initial values to $(3.10)$ at $x=0$ which converge to $k=-\sqrt{2 \omega}$ for $x \rightarrow \infty$, the slice of the stable manifold at $x=0$. The solution with $k(x=0)=k_{+}^{*}$ then converges to $k=\sqrt{2 \omega}$. Analogously, we define $W_{-}^{\mathrm{u}}=\left(k_{-}^{*},+\infty\right)$ as the set of initial values that converge to $k=\sqrt{2 \omega}$ for $x \rightarrow-\infty$, so that $k(x=0)=k_{-}^{*}$ is the unique initial value to the solution which converges to $k=-\sqrt{2 \omega}$ for $x=-\infty$. We set $\gamma=\sqrt{2 \omega} \geq 0$. It is not difficult to see that

$$
k_{+}^{*}=\gamma-\varepsilon \int_{0}^{\infty} G+\mathrm{O}\left(\varepsilon^{2}+\gamma^{2}\right), \quad k_{-}^{*}=-\gamma+\varepsilon \int_{-\infty}^{0} G+\mathrm{O}\left(\varepsilon^{2}+\gamma^{2}\right) .
$$

We find bounded solutions, whenever $k_{+}^{*}-k_{-}^{*} \geq 0$, that is, when

$$
2 \gamma \geq \varepsilon \int_{-\infty}^{\infty} G+\mathrm{O}\left(\varepsilon^{2}\right)
$$

If $\varepsilon \int G<0$, bounded solutions exist for all values of $\gamma$. If $\varepsilon \int G>0$, bounded solutions only exist for $\gamma \geq \varepsilon \int G / 2$. The phase portrait in extended phase space is as depicted in Figure 3.1. The construction of the bifurcation diagram can be adapted to the case where $G(x)=\mathrm{o}(1 / x)$, when the decay of $G$ exceeds the decay of $k$, using a variation-of-constant formula. From the heteroclinic bifurcation picture in Figure 3.1, one can readily infer the bifurcation diagram in Figure 1.2.

We close with a short phenomenological interpretation of these findings. For $\varepsilon<0$, incoming waves with wavenumber $k_{\infty}$ are transmitted across the inhomogeneity with a phase jump $-\varepsilon \int G / k$. For $\varepsilon>0$, the incoming waves are transmitted as long as their wavenumber is large enough, so that waves emitted by the source are pushed towards the inhomogeneity. When the wavenumber of the incoming waves is less or equal to the emitted wavenumber, an interface between the incoming waves and the waves emitted by the source is pushed away from the inhomogeneity so that the final state is the pure source. The latter scenario is the building block of the situation in two space-dimensions which we will address next in Section 3.3.

An interesting question arises when trying to understand this bifurcation from a path following point of view. Sources, as codimension-one heteroclinic orbits are robust and can be followed in parameters such as $\varepsilon$. While global path following results for heteroclinic orbits are not available, one can still try to continue the source ad hoc through $\varepsilon=0$. The natural continuation becomes apparent in the dual picture of eigenvalues of Schrödinger operators: the eigenvalue corresponding to the source disappears in the essential spectrum. It can however be continued as a zero of the analytic continuation of the pointwise resolvent, or the 

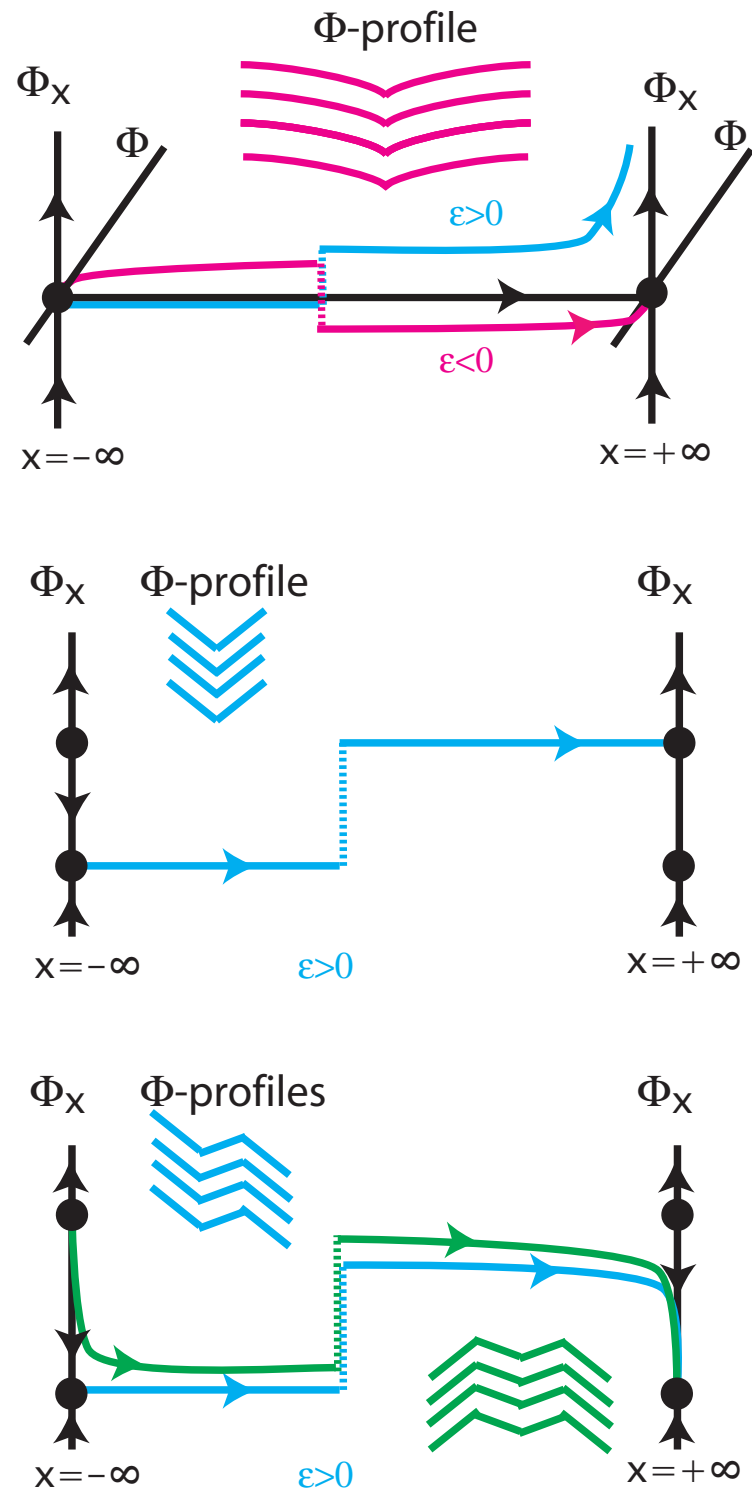

Figure 3.1: Schematic picture of the existence of sources and sinks in the eikonal approximation for small potentials. Phase portraits are in the phase space of wavenumber, vertical, space, horizontal and compactified. Phase is irrelevant and suppressed. In the first picture, we find contact defects, in the second, sources, and in the last picture sinks and transmission defects, constructed from sources and sinks. 
scattering function for $\varepsilon<0$. It then corresponds to a resonance pole, exhibiting pure exponential growth. In the ODE bifurcation picture for the eikonal equation, these resonance poles correspond to a heteroclinic connection between strong unstable and strong stable manifolds. The corresponding sinks separate two different kinds of sinks. For the first, regular type, with large enough incoming wavenumber, the wavenumber decreases monotonically along the sink. Sending in smaller wavenumbers, the profile changes to a non-monotone profile, where wavenumbers (and therefore group velocities) increase in magnitude while approaching the sink, before they decrease close to the sink and change sign. The maximal wavenumber attained by those sinks is the one selected by the resonance pole. In this sense, even though the source disappeared, the ghost of the source still selects a wavenumber in an intermediate range!

\subsection{Moving sources - conical and diffusive shock profiles}

We now consider the case of a moving inhomogeneity, or, equivalently, the system

$$
u_{t}=D \triangle u+c \varepsilon u_{x_{1}}+f(u)+\varepsilon^{2} g(\varepsilon x, u),
$$

where $x=\left(x_{1}, y\right) \in \mathbb{R} \times \mathbb{R}^{n-1}$. With (1.5), we find the eikonal equation in a comoving frame

$$
\Phi_{T}=d \Delta_{X} \Phi+c \Phi_{X_{1}}-\frac{1}{2} \Omega^{\prime \prime}\left|\nabla_{X} \Phi\right|^{2}+\bar{G}(X),
$$

with $X=\left(X_{1}, Y\right)=\varepsilon\left(x_{1}, y\right)$, and $T=\varepsilon^{2} t$. The transformations

$$
\Psi=\Phi+b x_{1}, \quad b=c / \Omega^{\prime \prime}, \quad \text { and } A=\mathrm{e}^{a \Psi}, \quad a=-2 d / \Omega^{\prime \prime},
$$

give

$$
A_{T}=d \Delta A-\frac{c^{2}}{2 \Omega^{\prime \prime}} A+\bar{G}(X) A .
$$

Coherent structures again correspond to eigenfunctions of the self-adjoint Schrödinger eigenvalue problem

$$
-\omega A=d \Delta A-\frac{c^{2}}{2 \Omega^{\prime \prime}} A+\bar{G}(X) A .
$$

If we adjust the frequency for the Doppler shift $\hat{\omega}:=\omega-\frac{c^{2}}{2 \Omega^{\prime \prime}}$, we recover the eigenvalue problem from the previous section. In particular, Proposition 3.1 gives wavenumber asymptotics

$$
\nabla \Phi=\gamma \frac{x}{|x|}-b e_{1}+\mathrm{O}\left(\frac{1}{|x|}\right)
$$

where $\gamma=\sqrt{-\hat{\omega}}, b$ was defined in (3.15), and $e_{1} \in \mathbb{R}^{n}$ denotes the unit vector in the direction of $x_{1}$. Level lines are radial where the phase gradient is perpendicular to $x$, that is, when $\gamma-b\left(e_{1}, x /|x|\right)=0$. This gives the typical sonic cone with opening angle $\vartheta=\arccos (\gamma / b)$; see also the numerical simulations in Figure 3.2.

A more typical scenario would be a source moving with speed $c=\mathrm{O}(1)$,

$$
u_{t}=D \Delta u+c u_{x_{1}}+f(u)+\varepsilon^{2} g\left(\varepsilon^{2} x_{1}, \varepsilon y, u\right) .
$$


We insert the Ansatz

$$
u(t, x)=u_{*}\left(\Phi(T, X)-\omega t ; \varepsilon \nabla_{X} \Phi(T, X)\right)+\varepsilon^{2} u_{1}(-\omega t, T, X) ; \quad X=\left(\varepsilon^{2} x_{1}, \varepsilon y\right), T=\varepsilon^{2} t,
$$

and find

$$
\Phi_{T}=d \Delta_{Y} \Phi+c \Phi_{X_{1}}-\frac{1}{2} \Omega^{\prime \prime}\left|\nabla_{Y} \Phi\right|^{2}+\bar{G}\left(X_{1}, Y\right)
$$

Transforming

$$
A=\mathrm{e}^{a \Phi}, \quad a=-2 d / \Omega^{\prime \prime},
$$

linearizes the equation which results in

$$
A_{T}=d \Delta_{Y} A+c A_{X_{1}}+\bar{G}\left(X_{1}, Y\right) A .
$$

Coherent structures are solutions to the heat equation

$$
-c A_{X_{1}}=d \Delta_{Y} A+\omega A+\bar{G}\left(X_{1}, Y\right) A .
$$

Removing the exponential growth $\mathrm{e}^{-\omega X_{1} / c}$, induced again by a Doppler shift, and rescaling $Y$ and $X_{1}$, we find

$$
A_{X_{1}}=\Delta_{Y} A+\bar{G}\left(X_{1}, Y\right) A .
$$

We focus on solutions with decay of the superimposed wave number, that is, decay of $\nabla(\log A)$, as $X_{1} \rightarrow-\infty$. This is equivalent to the shooting condition in the analysis of the preceding section, where we imposed a wavenumber $k$ at $-\infty$ and deduced wavenumber and phase jump at $+\infty$. Such solutions can be written in the form $A=1+B$ for a localized $B$, which solves the fixed point equation

$$
B\left(X_{1}, \cdot\right)=\int_{-\infty}^{X_{1}}\left(T\left(X_{1}-x_{1}\right) \bar{G}\left(x_{1}, \cdot\right)\left(1+B\left(x_{1}, \cdot\right)\right)\right) \mathrm{d} x_{1} .
$$

Here, $T(\xi)$ is the heat semigroup, given as a convolution operator

$$
(T(\xi) f)(y)=\frac{1}{\sqrt{4 \pi \xi}} \int_{\mathbb{R}} \mathrm{e}^{-\frac{\left(y-y^{\prime}\right)^{2}}{4 \xi}} f\left(y^{\prime}\right) \mathrm{d} y^{\prime} .
$$

It is easy to see that localization of $\bar{G}$, for instance $\bar{G} \in L^{1}$ implies that there exists a unique solution $B$ such that $B$ decays as $X_{1} \rightarrow-\infty$. For $X_{1} \rightarrow+\infty$, this unique solution will approach a self-similar solution to the heat equation with asymptotics

$$
B\left(X_{1}, Y\right)=B_{*} \frac{1}{\sqrt{4 \pi X_{1}}} \mathrm{e}^{-\frac{Y^{2}}{4 X_{1}}}\left(1+\mathrm{o}_{1 / X_{1}}(1)\right),
$$

in $L^{1} \cap L^{\infty}$. In particular, level lines are close to parabolas, $Y^{2}-\frac{1}{2} X_{1} \log X_{1}=$ const as $X_{1} \rightarrow \infty$. We note that these results should be valid only in an intermediate regime, until the next-order correction in the derivation of the modulation equation becomes relevant. We therefore expect a crossover at a finite, but large distance from a parabola to the conical sector described in Section 3.1.

The simulations in Figure 3.2 confirm these predictions in the setting of a reaction-diffusion system with a homogeneous oscillation. In particular, we observe the conical and parabolic profile for weak and strong drift, respectively. 

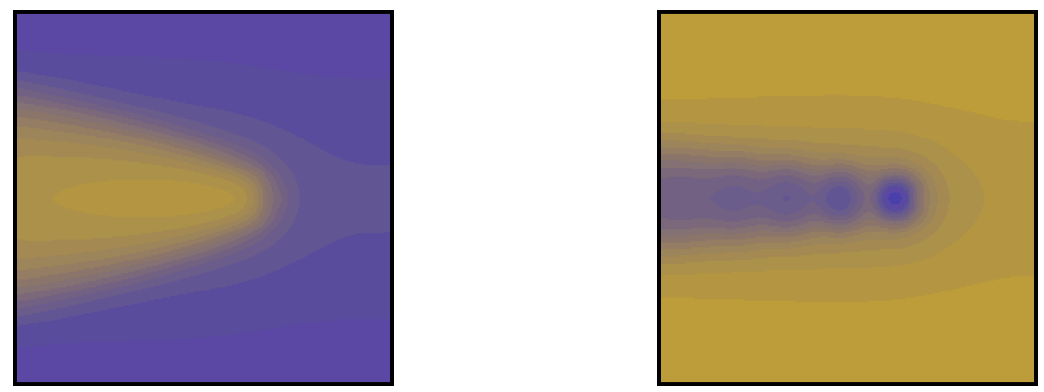

Figure 3.2: Simulations of (1.11), $\varepsilon=0.2$, with parameters from the introduction , and a horizontal drift $c=1.34$ in the left-hand and $c=2.68$ in the right hand picture. Plotted is the asymptotic state when starting with a spatially homogeneous initial condition. Click on the snapshots to see a movie of the time evolution.

\section{Discussion}

\subsection{Summary}

We proposed to study inhomogeneities in oscillatory media as an example for the creation and annihilation of coherent structures. Motivated by the one-dimensional case, we emphasized group velocities in the far field as the primary characteristic. In the examples we found, we were able to compute these group velocities. In the simplest, radial case, group velocities are radial and coherent structures are either sources, sinks, or contact structures. In two (or less) space dimensions, small inhomogeneities can create sources. Contact defects are always weak sinks, group velocities at finite but large distance point inwards. In more than two space dimensions, small potentials create weak sources or sinks, with group velocities converging to zero in the far field. In fact, the phase jump between the center and infinity is finite, so that typically only a finite number of rings are observed in physical space. Our study of moving inhomogeneities revealed more subtle effects. Group velocities may point outward only in a sector and inward along the complement. In analogy to the one-dimensional situation, we would refer to these structures as transmission defects, since waves both enter and leave a fixed neighborhood of the defect.

\section{$4.2 \quad$ Fluxes}

Instead of retaining only the sign of $\left(c_{\mathrm{g}}, x /|x|\right)$, that is, inward versus outward transport, we may look at the group velocity as a map from $S^{n-1}$ to $\mathbb{R}^{n}$, on large centered spheres. In most of our cases, this map converges as the size of the sphere tends to infinity. There are then various ways to extract quantitative information from this map in order to characterize the coherent structures. For instance, we may define the net flux $J$ associated with the phase of a coherent structure $\Phi$ as

$$
J(\Phi)=\lim _{R \rightarrow \infty}\left(R^{n-1} \operatorname{Vol}\left(S^{n-1}\right)\right)^{-1} \int_{|x|=R} j(x) \mathrm{d} \sigma, \quad \text { with } j(x)=\left(c_{\mathrm{g}}(x), x /|x|\right),
$$

where $\sigma$ is the $(n-1)$-dimensional Lebesgue surface element on $|x|=R \subset \mathbb{R}^{n}$. In addition to the distinction between sources, $J>0$, contact defects, $J=0$, and sinks $J<0, J$ also 
retains the strength of the source, so $J \sim \varepsilon$ in one space dimension, and $J \sim \mathrm{e}^{-1 / \varepsilon}$ in two space dimensions, where we neglected normalizing constants. This can be readily seen from our main results on the expansion for the wavenumber $k$ in the far field. Indeed, $c_{\mathrm{g}} \sim k$ since $\omega \sim k^{2}$, which immediately gives the expansion using Theorem 1 in the radial case and Proposition 3.1 in the case of the eikonal approximation; see also the remark at the end of Section 3.1. As a limit, $J$ does not retain the correction terms which distinguish between weak sources and sinks. For the transmission defects that we discussed in the case of slowly moving sources, the mean drift cancels in the integral so that we are lead to interpreting the case $\varepsilon M>0$ as a source rather than a transmission defect: transmission in the horizontal direction is superimposed by a source term, visible in particular in the vertical direction. For inhomogeneities moving with finite speed, it is not difficult to see that the limit actually vanishes: group velocities generated by the inhomogeneity decay almost everywhere.

\subsection{Degrees}

A more phenomenological classification would look at constant level lines of $\Phi$, or $u$, directly. We showed that level lines are expanding circles, with bounded and converging correction terms in case of anisotropic inhomogeneities. In case of moving inhomogeneities, we found cones and parabolas in the far field. Level lines need not form closed curves, as the case of spiral waves illustrates. In our case, however, it is not difficult to see that spirals cannot form. Indeed, one can define the topological degree of the defect as the degree $i_{\text {phase }}$ of the phase as a map from a large circle into a circle [25]. Since the phase is everywhere defined in our case of weak inhomogeneities, this map extends to a map from the disc into to the circle so that the degree vanishes. Note that fluxes do not distinguish between target patterns and spiral waves, while the topological degree does not distinguish between sources and sinks. Another degree $i_{\text {grad }}$ would be the Brouwer degree of $c_{\mathrm{g}}$. If $j>0$ pointwise (source), then $i_{\text {grad }}=1$,

and if $j<0$ pointwise, then $i_{\text {grad }}=-1$, regardless of $i_{\text {phase. }}$ For a plane wave, $i_{\text {grad }}=0$. Note however that $i_{\text {grad }}$ need not be defined for all coherent structures, since group velocities may vanish even in the far field.

\subsection{Stability}

Using the methods employed to prove existence, one can also track eigenvalues of the linearization. We conjecture that all coherent structures discussed here are stable. In the eikonal approximation, this can be seen after a Cole-Hopf transformation: solutions to the heat equation with source term approach the eigenfunction with the largest eigenvalue, exponentially when there is a spectral gap. For the reaction-diffusion systems, we expect that the methods developed in Section 2 should give spectral stability. We are not aware of nonlinear stability proofs for sources in reaction diffusion system; see however [7] for a related result for a transmission defect. 


\section{Appendix}

We prove Proposition 3.1. Our proof follows the ideas outlined in Section 2, setting up a dynamical systems framework where asymptotics are a consequence of expansions on stable and unstable manifolds. We rewrite the equation (3.6) as

$$
A_{r r}+\frac{1}{r} A_{r}+\frac{1}{r^{2}} A_{\varphi \varphi}+G(r, \varphi) A-\gamma^{2} A=0, \quad \omega=-\gamma^{2}
$$

in the form

$$
\begin{aligned}
& A_{r}=\mathcal{M} B \\
& B_{r}=\mathcal{M} A-\frac{\gamma^{2}}{r} \mathcal{M}^{-2} B-\mathcal{M}^{-1} G A,
\end{aligned}
$$

where $\mathcal{M}$ is the unbounded self-adjoint operator

$$
\mathcal{M}=\sqrt{-\frac{1}{r^{2}} \partial_{\varphi \varphi}+\gamma^{2}} \geq \gamma
$$

and $A, B \in L^{2}\left(S^{1}\right)$. We set $w_{ \pm}=A \pm B$. Since $\mathcal{M}$ is self-adjoint and positive, and the correction terms are bounded as operators, with norm $\mathrm{O}\left(r^{-1}\right)$, by the assumptions on $\bar{G}$, this equation possesses an exponential dichotomy [14]: there is $\Psi(r): L^{2} \rightarrow L^{2}, \Psi=\mathrm{O}\left(r^{-1}\right)$ so that any solution that is bounded as $r \rightarrow \infty$ satisfies $w_{+}=\Psi w_{-}$. We end up with an evolution equation for $w_{-}$which describes the asymptotics of any bounded solution:

$$
w_{-}^{\prime}=\mathcal{L}_{-}(r) w_{-}, \quad \mathcal{L}_{-}=-\mathcal{M}+\mathcal{N}, \text { with } \mathcal{N} \text { bounded, } \mathcal{N}=-\frac{\gamma^{2}}{2 r} \mathcal{M}^{-2}+\mathrm{O}\left(r^{-(1+\beta)}\right) .
$$

We start analyzing the asymptotics neglecting the bounded $\mathrm{O}\left(r^{-(1+\beta)}\right)$-terms contained in $\mathcal{N}$. The truncated equation is diagonal in Fourier modes $w_{-}=\sum_{k \in \mathbb{Z}} w_{k} \mathrm{e}^{\mathrm{i} k \varphi}$,

$$
\left(w_{-}^{k}\right)^{\prime}=\mathcal{L}_{-}^{k} w_{-}^{k}, \quad \mathcal{L}_{-}^{k}=-\gamma-\frac{1}{2 r}+\mathrm{O}\left(r^{-(1+\beta)}\right) .
$$

We next set $w_{k}:=\sqrt{r} \mathrm{e}^{\gamma r} w_{-}^{k}$, and find

$$
w_{k}^{\prime}=\left(\gamma-\sqrt{\frac{k^{2}}{r^{2}}+\gamma^{2}}\right) w_{k}+\frac{k^{2}}{2 r\left(k^{2}+\gamma^{2} r^{2}\right)} w_{k}+\mathrm{O}\left(r^{-(1+\beta)} w\right),
$$

or short,

$$
w_{k}^{\prime}=\frac{1}{r^{2}} \varphi\left(\frac{1}{r} ; k\right) w_{k}+\mathrm{O}\left(r^{-(1+\beta)} w\right) .
$$

Error terms are coupling all $w_{k}$, but are uniformly bounded as operators on $\ell^{2}$. Since the right-hand side is $\mathrm{O}\left(r^{-(1+\beta)}\right)$, we can introduce the compactified time variable $\tau=-1 /\left(\beta r^{\beta}\right)$ and find

$$
\dot{w}_{k}=\tau^{1-\beta} \varphi(\tau ; k) w_{k}+\mathrm{O}(w) .
$$

More precisely, we have

$$
\varphi(\tau ; k)=\tau^{1-\beta}\left(\gamma-\sqrt{\gamma^{2}+\tau^{2} k^{2}}\right)+\tau \frac{k}{2\left(k^{2} \tau^{2}+\gamma^{2}\right)}+\mathrm{O}\left(\tau^{-(1+\beta)} G(-1 / \tau)\right) .
$$


Here we assume $\beta \leq 1$ without loss of generality. The flow map from a fixed time $\tau=-1 / r_{0}$ to $\tau=0$ is readily verified to be bounded (and actually differentiable in $r_{0}$ ), using the explicit representation in Fourier modes and a variation-of-constant formula. This provides us with the desired limiting profile $w$, and, substituting back, with asymptotics for $w_{-}, w_{+}, u$, and $v$. The gradient estimates readily follow from the same argument, carried out in the space $\ell_{2}^{1}$, with $\sum_{k}\left|w_{k}\right|^{2}|k|^{2}<\infty$.

\section{References}

[1] S. Agmon. Lectures on exponential decay of solutions of second-order elliptic equations: bounds on eigenfunctions of $N$-body Schrödinger operators. Mathematical Notes, 29. Princeton University Press, 1982.

[2] R. Carmona and B. Simon. Pointwise bounds on eigenfunctions and wave packets in $N$-body quantum systems. V. Lower bounds and path integrals. Comm. Math. Phys. 80 (1981), 59-98.

[3] A. Doelman, B. Sandstede, A. Scheel, and G. Schneider. The dynamics of modulated wave trains. To appear in Memoirs of the Amer. Math. Soc.

[4] F. Dumortier. Techniques in the theory of local bifurcations: Blow-up, normal forms, nilpotent bifurcations, singular perturbations. In: "Bifurcations and Periodic Orbits of Vector Fields" (D. Schlomiuk, ed.). Kluwer, Dordrecht, NATO ASI Ser. C 408 (1993) $19-73$.

[5] D. Barkley. EZ-SPIRAL, A Code for Simulating Spiral Waves. HTTP://WWW.MATHS.WARWICK.AC.UK/ BARKLEY/SOFTWARE/EZ_SOFTWARE.HTML

[6] B. Blasius and L. Stone. Chaos and phase synchronization in ecological systems. Int. J. Bifurcation Chaos 10 (2000), 2361.

[7] T. Gallay, G. Schneider, and H. Uecker. Tansport of information near essentially unstable localized structures. Discrete Contin. Dyn. Syst. Ser. B 4 (2004), 349-390.

[8] G. Iooss and A. Mielke. Bifurcating time-periodic solutions of Navier-Stokes equations in infinite cylinders. J. Nonlinear Science 1 (1991), 107-146.

[9] R. Kapral and K. Showalter. Chemical Waves and Patterns. Kluwer Academic, Dordrecht, 1995.

[10] N. Kopell and L.N. Howard. Target patterns and horseshoes from a perturbed central-force problem: some temporally periodic solutions to reaction-diffusion equations. Stud. Appl. Math. 64(1981), 1-56.

[11] M. Krupa and P. Szmolyan. Extending slow manifolds near transcritical and pitchfork singularities. Nonlinearity 14 (2001) 1473-1491. 
[12] L.D. Landau and E. M. Lifshitz. Quantum mechanics: non-relativistic theory. Course of Theoretical Physics, Vol. 3. Pergamon Press, 1958.

[13] B.G. Pachpatte. Mathematical inequalities. North-Holland Mathematical Library, 67. Elsevier B. V., Amsterdam, 2005.

[14] D. Peterhof, B. Sandstede, and A. Scheel. Exponential dichotomies for solitary wave solutions of semilinear elliptic equations on infinite cylinders. J. Diff. Eqns. 140 (1997), 266-308.

[15] B. Sandstede and A. Scheel. Essential instability of pulses and bifurcations to modulated travelling waves Proc. Roy. Soc. Edinb. A 129 (1999), 1263-1290.

[16] B. Sandstede and A. Scheel. On the structure of spectra of modulated travelling waves. Math. Nachr. 232 (2001), 39-93.

[17] B. Sandstede and A. Scheel. Defects in oscillatory media: toward a classification. SIAM J. Appl. Dyn. Syst. 3 (2004), 1-68.

[18] B. Sandstede and A. Scheel. Evans function and blow-up methods in critical eigenvalue problems. Dyn. Cont. Discr. Dyn. Sys. 10 (2004), 941-964.

[19] B. Sandstede, A. Scheel, and C. Wulff. Bifurcations and dynamics of spiral waves. J. Nonlinear Science 9 (1999), 439-478.

[20] A. Scheel. Radially symmetric patterns of reaction-diffusion systems. Mem. Amer. Math. Soc. 165 (2003).

[21] B. Simon. The bound state of weakly coupled Schrödinger operators in one and two dimensions. Ann. Physics 97 (1976), 279-288.

[22] M. Stich and A.S. Mikhailov. Target patterns in two-dimensional heterogeneous oscillatory reaction-diffusion systems. Physica D 215 (2006), 38-45.

[23] F. Varela, J. P. Lachaux, E. Rodriguez, and J. Martinerie. The brainweb: phase synchronization and large-scale integration. Nat. Rev. Neurscience 2(2001), 229.

[24] A. T. Winfree. Electrical turbulence in three-dimensional heart muscle. Science 266 (1994), 1003-1006.

[25] A.T. Winfree. The geometry of biological time. Interdisciplinary Applied Mathematics, 12. Springer-Verlag, New York, 2001. 